\title{
Effect of the ionization source on the targeted analysis of Nickel and Vanadyl Porphyrins in crude oil
}

\author{
Juan S. Ramírez-Pradilla,${ }^{\dagger}$ Cristian Blanco-Tirado, ${ }^{\dagger}$ Marie-Hubert-Roux, ${ }^{\ddagger} \S$ Pierre
} Giusti,,${ }^{, a}$ Carlos Afonso ${ }^{\ddagger, \S}$ and Marianny Y. Combariza ${ }^{\dagger, *}$

†niversidad Industrial de Santander, Bucaramanga, Santander 680002, Colombia ¥CNRS/Université de Rouen, UMR 6014, COBRA, 76821 Mont Saint Aignan Cedex, France

§International Joint laboratory - iC2MC: Complex Matrices Molecular Characterization, 76700, Harfleur, France

"Total Refining and Chemicals, Total Research \& Technology Gonfreville, 76700, Harfleur, France

*Corresponding author: marianny@uis.edu.co

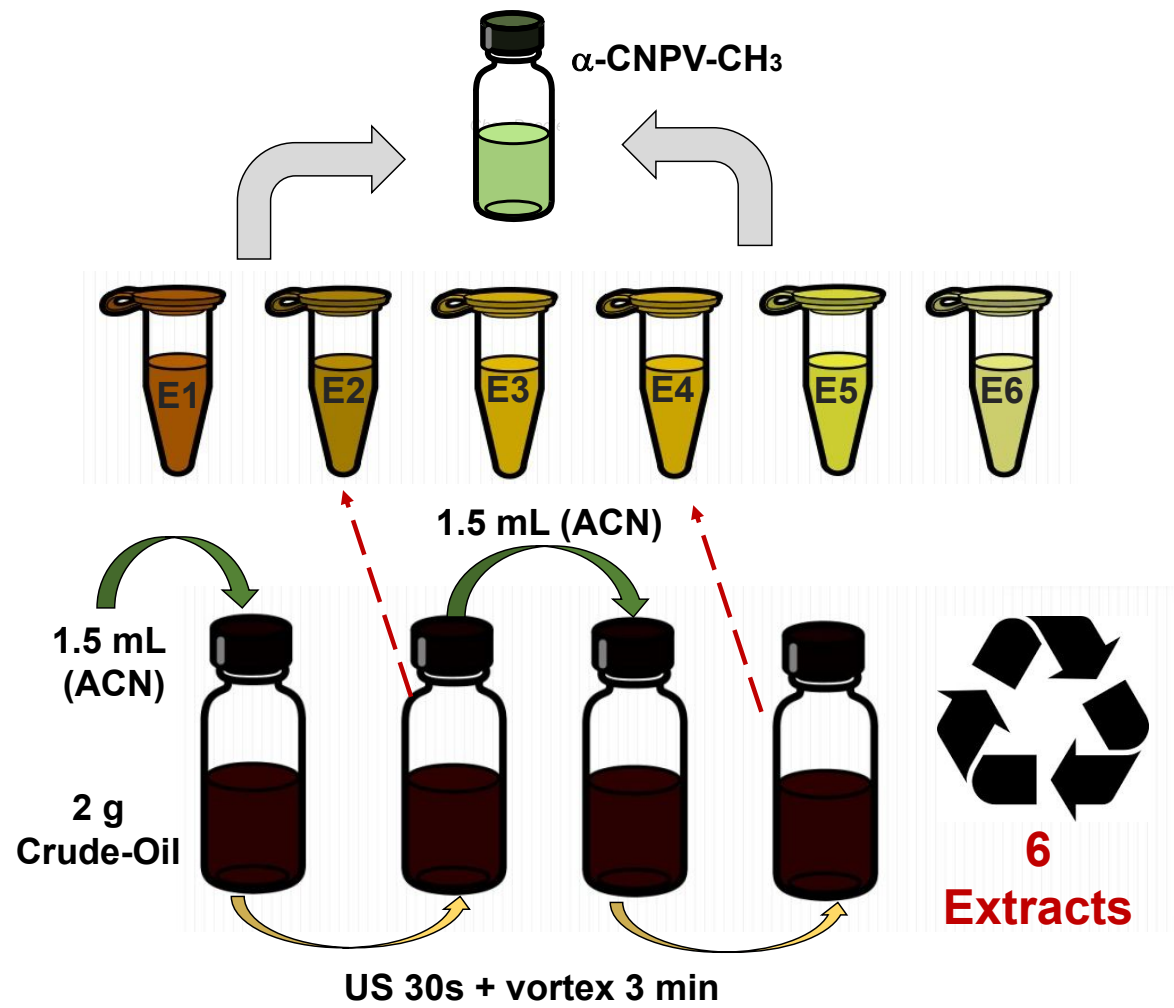

Figure S1. Liquid-Liquid consecutive extraction of acetonitrile:crude-oil system. 
Inductively coupled plasma - atomic emission spectroscopy measurements:

For ICP-AES analysis an iCAP-6500 Radial ICP mass spectrometer (Thermo Fisher Scientific, Waltham, MA) was used. The ACN extract obtained after liquid-liquid extraction from crude-oil were dried and weighted. Blank oil HB75 (Conostan, Analysis certificate $<0.02 \mathrm{ppm}$ for $\mathrm{Ni}$ and $<0.03 \mathrm{ppm}$ for $\mathrm{V}$ ) and xylene (VWR, Normapur min 99.2\%) were added in proportion to be in same conditions as calibration samples. Instrument conditions: RF power $1400 \mathrm{~W}$, plasma gas flow rate $12 \mathrm{~L} . \mathrm{min}^{-1}$, nebulizer gas flow rate, $0.40 \mathrm{~L}^{\mathrm{min}}{ }^{-1}$, auxiliary gas flow rate $1.5 \mathrm{~L} \mathrm{~min}^{-1}$, Yttrium was used as internal standard. Wavelengths; Ni: 221.647 and $231.604 \mathrm{~nm}$; V: 292.492 and $309.311 \mathrm{~nm} ; \mathrm{Y}: 224.396$ and $360.073 \mathrm{~nm}$. A concentration of $42 \mathrm{mg} / \mathrm{kg}$ and $77 \mathrm{mg} / \mathrm{kg}$ of nickel and vanadium, respectively, was found in the crude oil. The sum of consecutive ACN fractions give $4.8 \%$ and $8.9 \%$ of the total concentration of nickel and vanadium, respectively.

Table S1. Concentration of nickel and vanadium in ppm found in extracts E1 to E6 by ICP-OES

\begin{tabular}{|c|c|c|}
\hline Extract & $\mathrm{Ni}(\mathrm{mg} / \mathrm{kg})$ & $\mathrm{V}(\mathrm{mg} / \mathrm{kg})$ \\
\hline $\mathrm{E}_{1}$ & 9.9 & 35.2 \\
\hline $\mathrm{E}_{2}$ & 11.2 & 39.3 \\
\hline $\mathrm{E}_{3}$ & 9.4 & 37.8 \\
\hline $\mathrm{E}_{4}$ & 6.6 & 34.9 \\
\hline $\mathrm{E}_{5}$ & 16.4 & 46.8 \\
\hline $\mathrm{E}_{6}$ & 28.2 & 61.10 \\
\hline
\end{tabular}




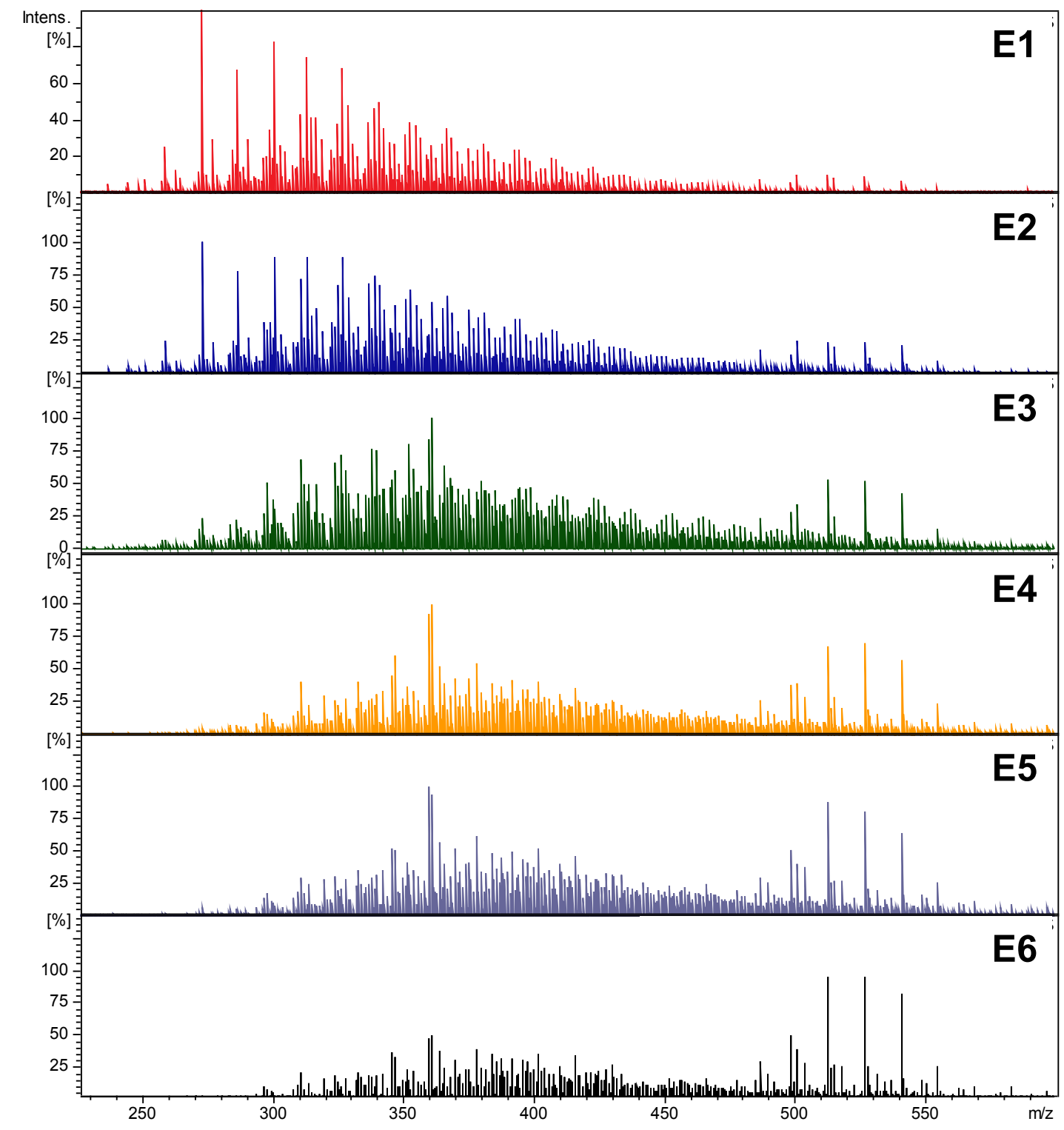

Figure S2. ET-MALDI-TOF-(+) spectra corresponding to each extract $+\alpha-\mathrm{CNPV}-\mathrm{CH}_{3}$ matrix. $E_{6}$ fraction showed the highest intensity of signals corresponding to petroporphyrins. 


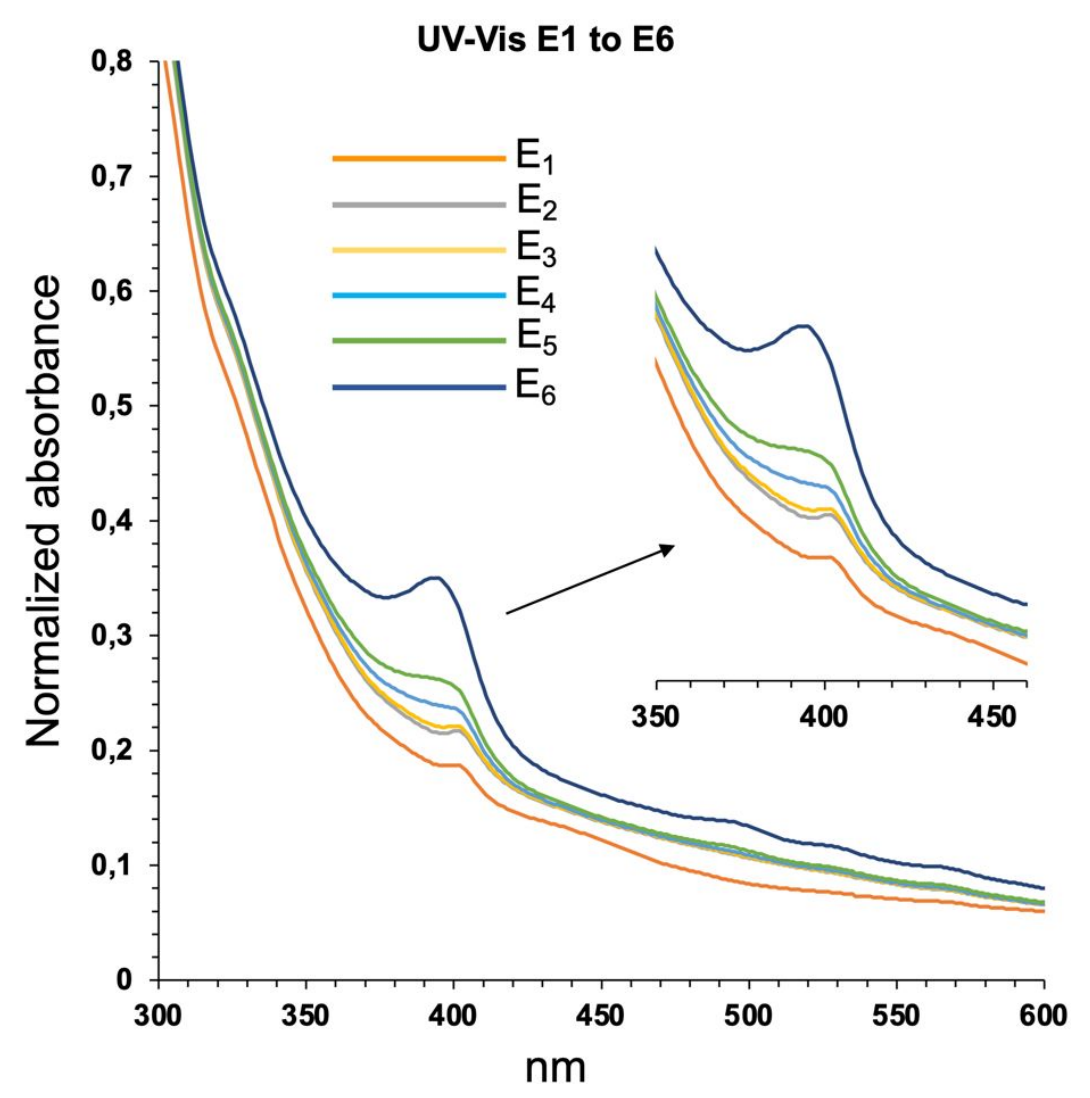

Figure S3. UV-Vis measurements for extracts $E_{1}$ to $E_{6}$. Insets shows the soret band associated with the presence of petroporphyirins.
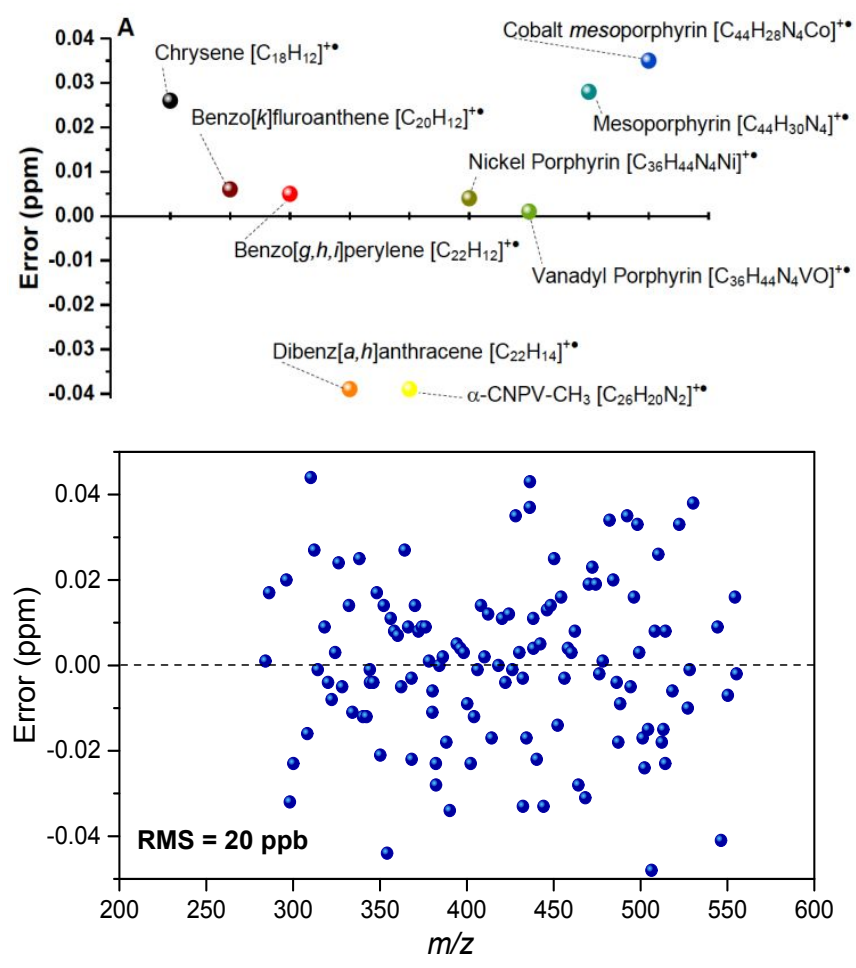
Figure S4. (A) External calibration using PAH class compounds and metalloporphyrins. (B) Internal calibration RMSE for extract $\mathrm{E}_{6}$ following the $\mathrm{CH}^{-\mathrm{R}}$ compound class.

ESI-(+)-FTICR experiment of $E_{6}$ : the extract $E_{6}$ was diluted 10 times in acetonitrile and doped with $1 \%$ of formic acid. For ESI-(+), solution was infused into mass spectrometer using a flow rate of $120 \mu \mathrm{L} \mathrm{h}^{-1}$. The ESI conditions were set as follows: capillary voltage 4500V; source temperature $200^{\circ} \mathrm{C}$; desolvation gas flow 4 L.min ${ }^{-1}$; nebulizer pressure 1 bar; quadrupole lower cutoff 100 and TOF $0.8 \mathrm{~ms}$. The mass spectrum was acquired with size $16 \mathrm{M}$ over a mass range of $\mathrm{m} / \mathrm{z} 92-1000$ with a $1.08 \mathrm{~s}$ transient length. The number of scans averaged was 100 . The accumulation time was set to $0.1 \mathrm{~s}$. The broadband spectrum is show in figure $\mathrm{S} 5$.

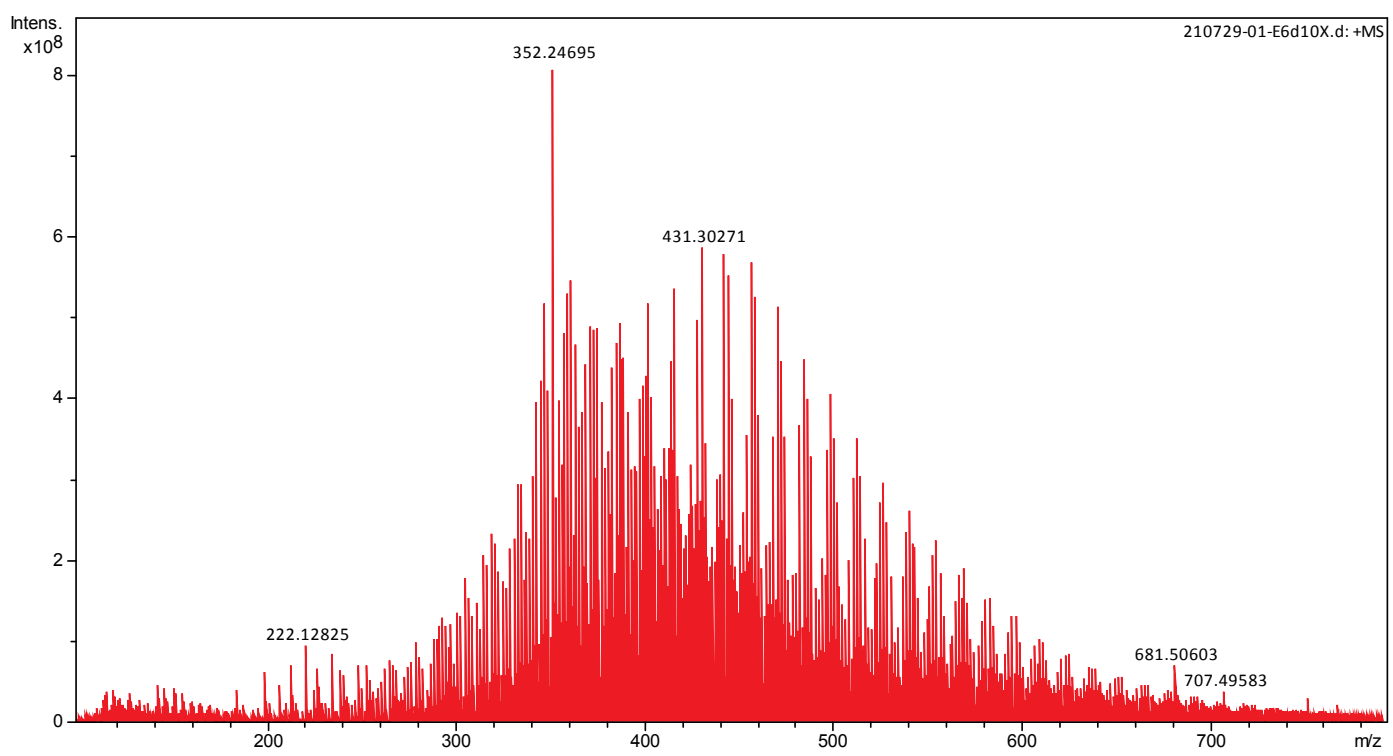




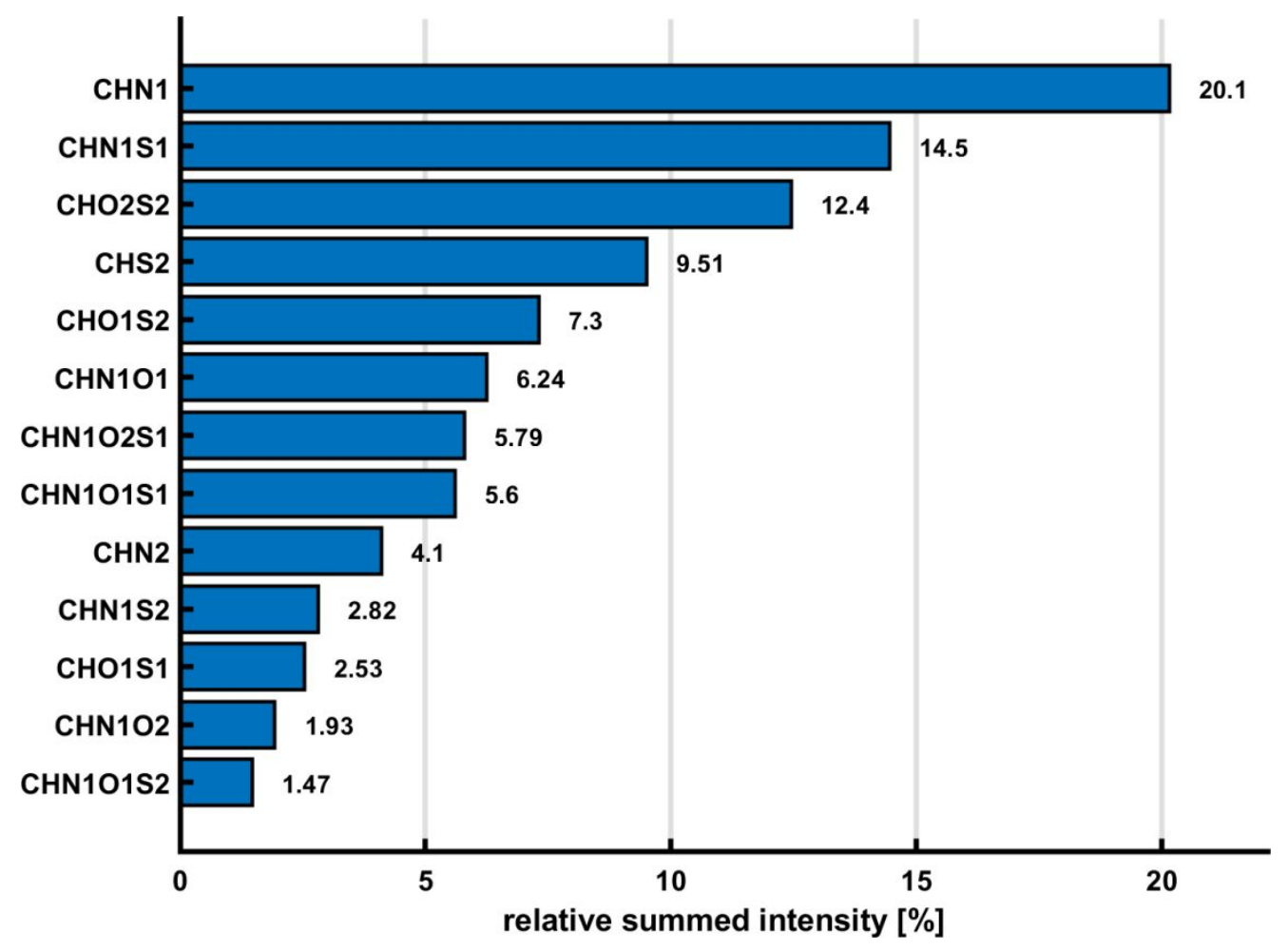

Figure S5. ESI-(+) spectrum for extract $\mathrm{E}_{6}$ with the respective heteroatom class distribution 


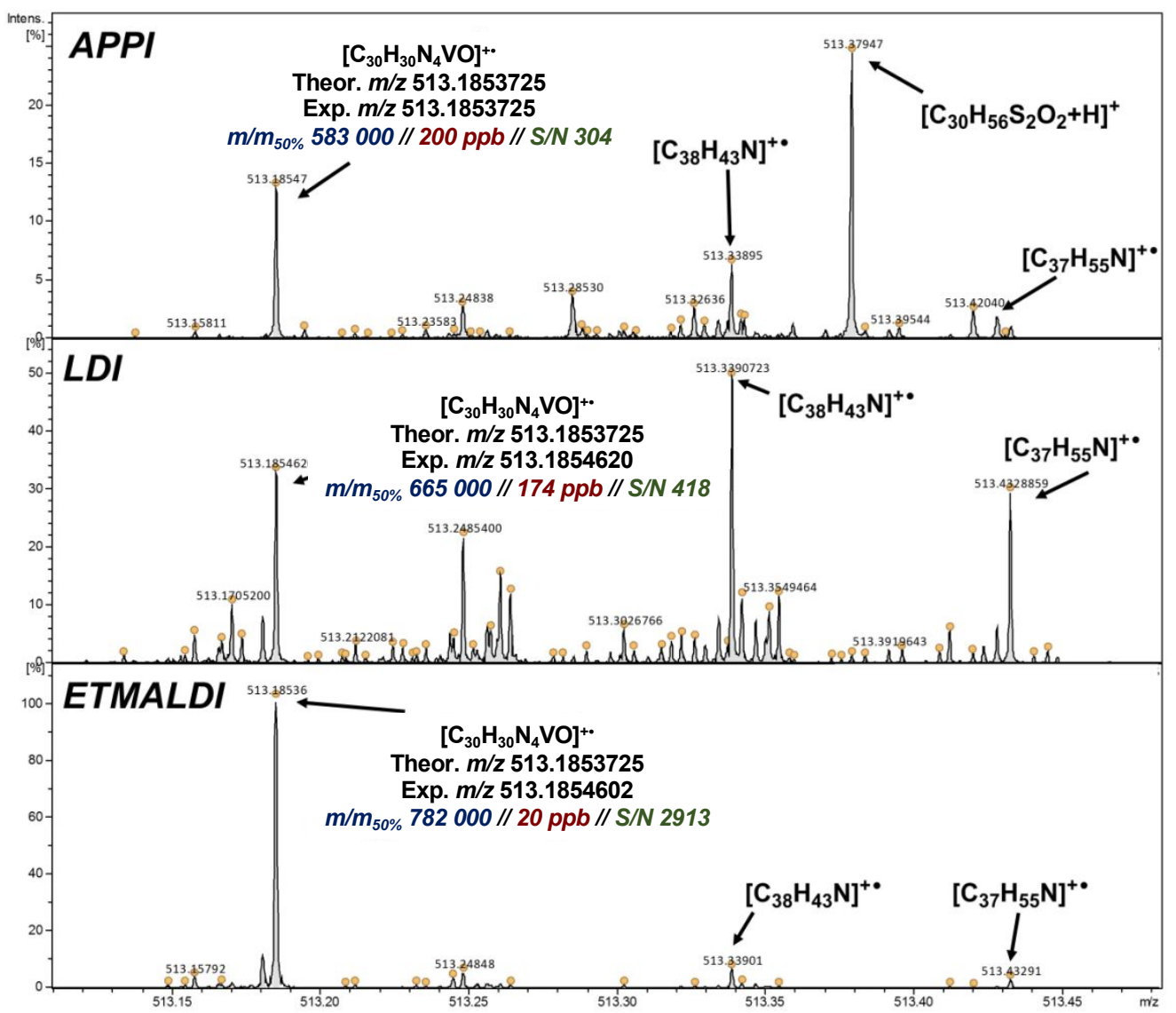

Figure S6. Expanded-scale view of E6 APPI-(+), LDI-(+), and ET-MALDI-(+) mass spectra at $m / z 513$ showing $S / N$ ratios, mass accuracy, and resolution for the vanadyl porphyrin $\left[\mathrm{C}_{30} \mathrm{H}_{30} \mathrm{~N}_{4} \mathrm{OV}\right]^{+\cdot}$ signal 
Table S2. Extension of figure 5 data for ET-MALDI, APPI and LDI in a zoomed region of $\mathrm{m} / \mathrm{z} 500$ to 600

\section{ET-MALDI Zoom 500 - 506}

\begin{tabular}{|c|c|c|c|c|}
\hline & {$\left[\mathrm{C}_{29} \mathrm{H}_{30} \mathrm{~N}_{4} \mathrm{VO}\right]^{+*}$} & {$\left[\mathrm{C}_{29} \mathrm{H}_{30} \mathrm{~N}_{3}{ }^{15} \mathrm{~N}_{1} \mathrm{VO}\right]^{+*}$} & {$\left[\mathrm{C}_{29}{ }^{13} \mathrm{C}_{1} \mathrm{H}_{30} \mathrm{~N}_{4} \mathrm{VO}\right]^{+\cdot}$} & {$\left[\mathrm{C}_{27}{ }^{13} \mathrm{C}_{2} \mathrm{H}_{30} \mathrm{~N}_{4} \mathrm{VO}\right]^{+*}$} \\
\hline Theor. mass & 501.185372 & 502.1824451 & 502.1887274 & 503.1920822 \\
\hline $\mathrm{S} / \mathrm{N}$ & 143.2 & 18.8 & 53.0 & 28.7 \\
\hline Accuracy & $32 \mathrm{ppb}$ & $75 \mathrm{ppb}$ & $14 \mathrm{ppb}$ & $18 \mathrm{ppb}$ \\
\hline & {$\left[\mathrm{C}_{30} \mathrm{H}_{30} \mathrm{~N}_{4} \mathrm{Ni}^{]^{+*}}\right.$} & {$\left[\mathrm{C}_{29}{ }^{13} \mathrm{C}_{1} \mathrm{H}_{30} \mathrm{~N}_{4} \mathrm{Ni}^{+{ }^{+*}}\right.$} & {$\left[\mathrm{C}_{30} \mathrm{H}_{30} \mathrm{~N}_{4}{ }^{60} \mathrm{Ni}\right]^{+\bullet}$} & {$\left[\mathrm{C}_{28}{ }^{13} \mathrm{C}_{2} \mathrm{H}_{30} \mathrm{~N}_{4} \mathrm{Ni}^{+{ }^{+}}\right.$} \\
\hline Theor. mass & 504.1818413 & 505.1851961 & 506.1772848 & 506.1885510 \\
\hline $\mathrm{S} / \mathrm{N}$ & 200.0 & 65.4 & 86.5 & 17.9 \\
\hline Accuracy & $-20 \mathrm{ppb}$ & $27 \mathrm{ppb}$ & $22 \mathrm{ppb}$ & $51 \mathrm{ppb}$ \\
\hline
\end{tabular}

\section{APPI Zoom 500 - 506}

\begin{tabular}{|c|c|c|c|c|}
\hline & {$\left[\mathrm{C}_{29} \mathrm{H}_{30} \mathrm{~N}_{4} \mathrm{VO}\right]^{+\bullet}$} & {$\left[\mathrm{C}_{29} \mathrm{H}_{30} \mathrm{~N}_{3}{ }^{15} \mathrm{~N}_{1} \mathrm{VO}\right]^{+\bullet}$} & {$\left[\mathrm{C}_{29}{ }^{13} \mathrm{C}_{1} \mathrm{H}_{30} \mathrm{~N}_{4} \mathrm{VO}\right]^{+\cdot}$} & {$\left[\mathrm{C}_{27}{ }^{13} \mathrm{C}_{2} \mathrm{H}_{30} \mathrm{~N}_{4} \mathrm{VO}\right]^{+\bullet}$} \\
\hline Theor. mass & 501.185372 & 502.1824451 & 502.1887274 & 503.1920822 \\
\hline S/N & 78.2 & 27.5 & 22.3 & Not observed \\
\hline Accuracy & $30 \mathrm{ppb}$ & $190 \mathrm{ppb}$ & $49 \mathrm{ppb}$ & Not observed \\
\hline & {$\left[\mathrm{C}_{30} \mathrm{H}_{30} \mathrm{~N}_{4} \mathrm{Ni}^{+\bullet}\right.$} & {$\left[\mathrm{C}_{29}{ }^{13} \mathrm{C}_{1} \mathrm{H}_{30} \mathrm{~N}_{4} \mathrm{Ni}^{+\cdot}\right.$} & {$\left[\mathrm{C}_{30} \mathrm{H}_{30} \mathrm{~N}_{4}{ }^{60} \mathrm{Ni}^{+\bullet}\right.$} & {$\left[\mathrm{C}_{28}{ }^{13} \mathrm{C}_{2} \mathrm{H}_{30} \mathrm{~N}_{4} \mathrm{Ni}^{+\bullet}\right.$} \\
\hline Theor. mass & 504.1818413 & 505.1851961 & 506.1772848 & 506.1885510 \\
\hline S/N & 35.4 & 10.9 & 12.7 & Not observed \\
\hline Accuracy & $-180 \mathrm{ppb}$ & $-183 \mathrm{ppb}$ & $-274 \mathrm{ppb}$ & Not observed \\
\hline
\end{tabular}

\section{LDI Zoom 500 - 506}

\begin{tabular}{|c|c|c|c|c|}
\hline & {$\left[\mathrm{C}_{29} \mathrm{H}_{30} \mathrm{~N}_{4} \mathrm{VO}\right]^{+\bullet}$} & {$\left[\mathrm{C}_{29} \mathrm{H}_{30} \mathrm{~N}_{3}{ }^{15} \mathrm{~N}_{1} \mathrm{VO}\right]^{+\bullet}$} & {$\left[\mathrm{C}_{29}{ }^{13} \mathrm{C}_{1} \mathrm{H}_{30} \mathrm{~N}_{4} \mathrm{VO}\right]^{+\bullet}$} & {$\left[\mathrm{C}_{27}{ }^{13} \mathrm{C}_{2} \mathrm{H}_{30} \mathrm{~N}_{4} \mathrm{VO}^{+\bullet}\right.$} \\
\hline Theor. mass & 501.185372 & 502.1824451 & 502.1887274 & 503.1920822 \\
\hline S/N & 78.2 & Not observed & 12.1 & Not observed \\
\hline Accuracy & $67 \mathrm{ppb}$ & Not observed & $180 \mathrm{ppb}$ & Not observed \\
\hline & {$\left[\mathrm{C}_{30} \mathrm{H}_{30} \mathrm{~N}_{4} \mathrm{Ni}^{+\bullet}\right.$} & {$\left[\mathrm{C}_{29}{ }^{13} \mathrm{C}_{1} \mathrm{H}_{30} \mathrm{~N}_{4} \mathrm{Ni}\right]^{+\bullet}$} & {$\left[\mathrm{C}_{30} \mathrm{H}_{30} \mathrm{~N}_{4}{ }^{60} \mathrm{Ni}^{+\bullet}\right.$} & {$\left[\mathrm{C}_{28}{ }^{13} \mathrm{C}_{2} \mathrm{H}_{30} \mathrm{~N}_{4} \mathrm{Ni}^{+\bullet}\right.$} \\
\hline Theor. mass & 504.1818413 & 505.1851961 & 506.1772848 & 506.1885510 \\
\hline S/N & 52.2 & 12.7 & 22.6 & Not observed \\
\hline Accuracy & $-223 \mathrm{ppb}$ & $-173 \mathrm{ppb}$ & $-294 \mathrm{ppb}$ & Not observed \\
\hline
\end{tabular}

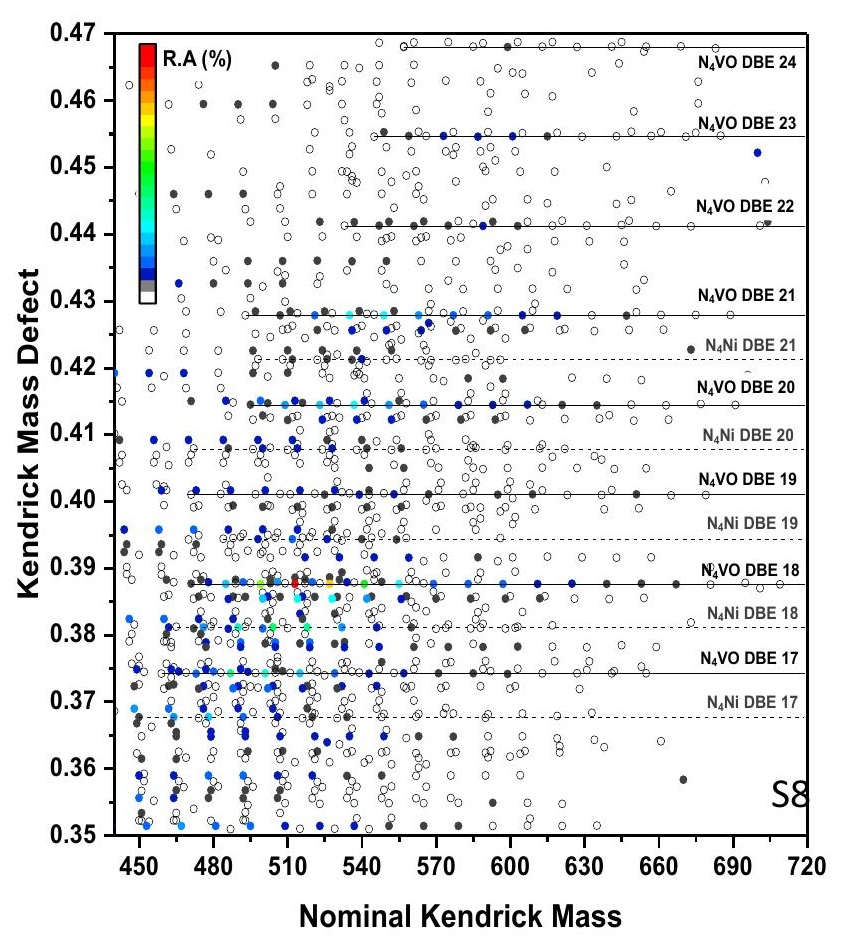




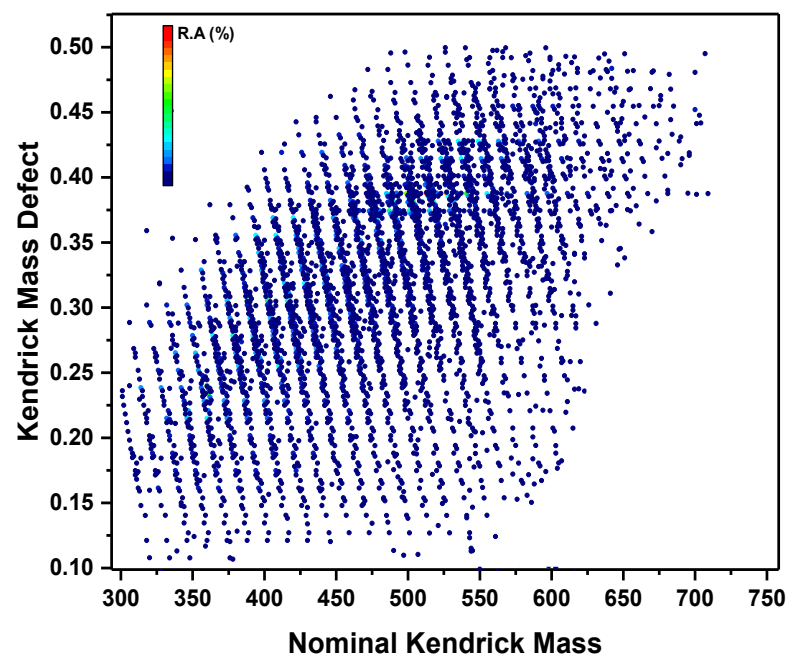

Figure S7. Kendrick Mass Defect vs.

Nominal Kendrick Mass plots used for the identification of porphyrin homologous series present in $\mathrm{E}_{6}$. Left: full compositional space of sample E6. Right: Zoom-in of the region with the highest $\mathrm{RA} \%$.

Table S1. Vanadyl Porphyrin distribution in fraction $E_{6}$

\begin{tabular}{ccccc} 
KMD = 0.37425 & \multicolumn{2}{c}{ Vanadyl Porphyrins “DBE = 17”” } & \multicolumn{2}{c}{ RMS = 27 ppb } \\
\hline $\begin{array}{c}\text { Molecular } \\
\text { Formula }\end{array}$ & $\begin{array}{c}\text { Experimental } \\
\mathrm{m} / \mathbf{z}\end{array}$ & $\begin{array}{c}\text { Theoretical } \\
\text { m/z }\end{array}$ & $\begin{array}{c}\text { Mass } \\
\text { accuracy } \\
\text { ppb }\end{array}$ & $\begin{array}{c}\text { Relative } \\
\text { Abundance }\end{array}$ \\
\hline $\mathrm{C}_{24} \mathrm{H}_{20} \mathrm{~N}_{4} \mathrm{VO}$ & 431.107107 & 431.107122 & 34 & 0.37 \\
$\mathrm{C}_{25} \mathrm{H}_{22} \mathrm{~N}_{4} \mathrm{VO}$ & 445.122758 & 445.122772 & 33 & 1.61 \\
$\mathrm{C}_{26} \mathrm{H}_{24} \mathrm{~N}_{4} \mathrm{VO}$ & 459.138408 & 459.138422 & 32 & 10.78 \\
$\mathrm{C}_{27} \mathrm{H}_{26} \mathrm{~N}_{4} \mathrm{VO}$ & 473.154065 & 473.154072 & 15 & 30.98 \\
$\mathrm{C}_{28} \mathrm{H}_{28} \mathrm{~N}_{4} \mathrm{VO}$ & 487.169704 & 487.169722 & 38 & 61.75 \\
$\mathrm{C}_{29} \mathrm{H}_{30} \mathrm{~N}_{4} \mathrm{VO}$ & 501.185356 & 501.185373 & 33 & 65.80 \\
$\mathrm{C}_{30} \mathrm{H}_{32} \mathrm{~N}_{4} \mathrm{VO}$ & 515.201010 & 515.201023 & 24 & 44.20 \\
$\mathrm{C}_{31} \mathrm{H}_{34} \mathrm{~N}_{4} \mathrm{VO}$ & 529.216669 & 529.216673 & 7 & 23.80 \\
$\mathrm{C}_{32} \mathrm{H}_{36} \mathrm{~N}_{4} \mathrm{VO}$ & 543.232321 & 543.232323 & 3 & 15.07 \\
$\mathrm{C}_{33} \mathrm{H}_{38} \mathrm{~N}_{4} \mathrm{VO}$ & 557.247963 & 557.247973 & 18 & 7.67 \\
$\mathrm{C}_{34} \mathrm{H}_{40} \mathrm{~N}_{4} \mathrm{VO}$ & 571.263620 & 571.263623 & 4 & 4.94 \\
$\mathrm{C}_{35} \mathrm{H}_{42} \mathrm{~N}_{4} \mathrm{VO}$ & 585.279278 & 585.279273 & -10 & 4.51 \\
$\mathrm{C}_{36} \mathrm{H}_{44} \mathrm{~N}_{4} \mathrm{VO}$ & 599.294925 & 599.294923 & -3 & 3.54 \\
$\mathrm{C}_{37} \mathrm{H}_{46} \mathrm{~N}_{4} \mathrm{VO}$ & 613.310589 & 613.310573 & -27 & 1.87 \\
$\mathrm{C}_{38} \mathrm{H}_{48} \mathrm{~N}_{4} \mathrm{VO}$ & 627.326249 & 627.326223 & -41 & 1.23 \\
$\mathrm{C}_{39} \mathrm{H}_{50} \mathrm{~N}_{4} \mathrm{VO}$ & 641.341883 & 641.341873 & -15 & 1.30 \\
$\mathrm{C}_{40} \mathrm{H}_{52} \mathrm{~N}_{4} \mathrm{VO}$ & 655.357556 & 655.357523 & -5 & 0.93 \\
$\mathrm{C}_{41} \mathrm{H}_{54} \mathrm{~N}_{4} \mathrm{VO}$ & 669.373183 & 669.373173 & -15 & 0.58
\end{tabular}




\begin{tabular}{|c|c|c|c|c|}
\hline $\mathrm{C}_{42} \mathrm{H}_{56} \mathrm{~N}_{4} \mathrm{VO}$ & 683.388844 & 683.388823 & -30 & 0.39 \\
\hline $\mathrm{C}_{43} \mathrm{H}_{58} \mathrm{~N}_{4} \mathrm{VO}$ & 697.404503 & 697.404473 & -42 & 0.29 \\
\hline $\mathrm{KMD}=0.38766$ & \multicolumn{3}{|c|}{ Vanadyl Porphyrins “DBE = 18” } & RMS = 38 ppb \\
\hline $\begin{array}{c}\text { Molecular } \\
\text { Formula } \\
\end{array}$ & $\begin{array}{c}m / z \\
\text { observed }\end{array}$ & $\begin{array}{c}m / z \\
\text { calculated }\end{array}$ & $\begin{array}{l}\text { Mass Error } \\
\text { ppb }\end{array}$ & $\begin{array}{c}\text { Relative } \\
\text { Abundance }\end{array}$ \\
\hline $\mathrm{C}_{23} \mathrm{H}_{16} \mathrm{~N}_{4} \mathrm{VO}$ & 415.075815 & 415.075822 & 18 & 0.13 \\
\hline $\mathrm{C}_{24} \mathrm{H}_{18} \mathrm{~N}_{4} \mathrm{VO}$ & 429.091461 & 429.091472 & 26 & 0.43 \\
\hline $\mathrm{C}_{25} \mathrm{H}_{20} \mathrm{~N}_{4} \mathrm{VO}$ & 443.107115 & 443.107122 & 17 & 1.44 \\
\hline $\mathrm{C}_{26} \mathrm{H}_{22} \mathrm{~N}_{4} \mathrm{VO}$ & 457.122762 & 457.122772 & 22 & 6.40 \\
\hline $\mathrm{C}_{27} \mathrm{H}_{24} \mathrm{~N}_{4} \mathrm{VO}$ & 471.138412 & 471.138422 & 21 & 17.96 \\
\hline $\mathrm{C}_{28} \mathrm{H}_{26} \mathrm{~N}_{4} \mathrm{VO}$ & 485.154071 & 485.154072 & 4 & 39.27 \\
\hline $\mathrm{C}_{29} \mathrm{H}_{28} \mathrm{~N}_{4} \mathrm{VO}$ & 499.169714 & 499.169722 & 16 & 76.89 \\
\hline $\mathrm{C}_{30} \mathrm{H}_{30} \mathrm{~N}_{4} \mathrm{VO}$ & 513.18537 & 513.185373 & 5 & 100 \\
\hline $\mathrm{C}_{31} \mathrm{H}_{32} \mathrm{~N}_{4} \mathrm{VO}$ & 527.201013 & 527.201023 & 19 & 93.60 \\
\hline $\mathrm{C}_{32} \mathrm{H}_{34} \mathrm{~N}_{4} \mathrm{VO}$ & 541.216651 & 541.216673 & 39 & 68.67 \\
\hline $\mathrm{C}_{33} \mathrm{H}_{36} \mathrm{~N}_{4} \mathrm{VO}$ & 555.232315 & 555.232323 & 14 & 28.50 \\
\hline $\mathrm{C}_{34} \mathrm{H}_{38} \mathrm{~N}_{4} \mathrm{VO}$ & 569.247971 & 569.247973 & 3 & 13.95 \\
\hline $\mathrm{C}_{35} \mathrm{H}_{40} \mathrm{~N}_{4} \mathrm{VO}$ & 583.263637 & 583.263623 & -25 & 10.15 \\
\hline $\mathrm{C}_{36} \mathrm{H}_{42} \mathrm{~N}_{4} \mathrm{VO}$ & 597.27929 & 597.279273 & -28 & 7.31 \\
\hline $\mathrm{C}_{37} \mathrm{H}_{44} \mathrm{~N}_{4} \mathrm{VO}$ & 611.294933 & 611.294923 & -16 & 4.04 \\
\hline $\mathrm{C}_{38} \mathrm{H}_{46} \mathrm{~N}_{4} \mathrm{VO}$ & 625.310602 & 625.310573 & -47 & 2.50 \\
\hline $\mathrm{C}_{39} \mathrm{H}_{48} \mathrm{~N}_{4} \mathrm{VO}$ & 639.326242 & 639.326223 & -29 & 2.65 \\
\hline $\mathrm{C}_{40} \mathrm{H}_{50} \mathrm{~N}_{4} \mathrm{VO}$ & 653.341872 & 653.341873 & 1 & 2.11 \\
\hline $\mathrm{C}_{41} \mathrm{H}_{52} \mathrm{~N}_{4} \mathrm{VO}$ & 667.357525 & 667.357523 & -3 & 1.29 \\
\hline $\mathrm{C}_{42} \mathrm{H}_{54} \mathrm{~N}_{4} \mathrm{VO}$ & 681.373212 & 681.373173 & -57 & 0.78 \\
\hline $\mathrm{C}_{43} \mathrm{H}_{56} \mathrm{~N}_{4} \mathrm{VO}$ & 695.38887 & 695.388823 & -67 & 0.62 \\
\hline $\mathrm{C}_{44} \mathrm{H}_{58} \mathrm{~N}_{4} \mathrm{VO}$ & 709.404504 & 709.404473 & -43 & 0.48 \\
\hline $\mathrm{C}_{45} \mathrm{H}_{60} \mathrm{~N}_{4} \mathrm{VO}$ & 723.420214 & 723.420123 & -125 & 0.35 \\
\hline $\mathrm{C}_{46} \mathrm{H}_{62} \mathrm{~N}_{4} \mathrm{VO}$ & 737.435782 & 737.435774 & -12 & 0.24 \\
\hline $\mathrm{C}_{47} \mathrm{H}_{64} \mathrm{~N}_{4} \mathrm{VO}$ & 751.451466 & 751.451424 & -57 & 0.15 \\
\hline
\end{tabular}

\begin{tabular}{ccccc} 
KMD = 0.40104 & \multicolumn{2}{c}{ Vanadyl Porphyrins “DBE = 19" } & \multicolumn{2}{c}{ RMS = 55 ppb } \\
\hline Molecular & $\begin{array}{c}\mathrm{m} / \mathbf{z} \\
\text { observed }\end{array}$ & $\begin{array}{c}\mathrm{m} / \mathbf{z} \\
\text { calculated }\end{array}$ & $\begin{array}{c}\text { Mass Error } \\
\text { ppb }\end{array}$ & $\begin{array}{c}\text { Relative } \\
\text { Abundance }\end{array}$ \\
\hline Formula & 427.075818 & 427.075822 & 10 & 0.53 \\
$\mathrm{C}_{24} \mathrm{H}_{16} \mathrm{~N}_{4} \mathrm{VO}$ & 441.091469 & 441.091472 & 7 & 1.51 \\
$\mathrm{C}_{25} \mathrm{H}_{18} \mathrm{~N}_{4} \mathrm{VO}$ & 455.107106 & 455.107122 & 37 & 5.70 \\
$\mathrm{C}_{26} \mathrm{H}_{20} \mathrm{~N}_{4} \mathrm{VO}$ & & &
\end{tabular}




\begin{tabular}{lcccc}
$\mathrm{C}_{27} \mathrm{H}_{22} \mathrm{~N}_{4} \mathrm{VO}$ & 469.122773 & 469.122772 & -2 & 12.38 \\
$\mathrm{C}_{28} \mathrm{H}_{24} \mathrm{~N}_{4} \mathrm{VO}$ & 483.13842 & 483.138422 & 4 & 17.01 \\
$\mathrm{C}_{29} \mathrm{H}_{26} \mathrm{~N}_{4} \mathrm{VO}$ & 497.154067 & 497.154072 & 10 & 18.07 \\
$\mathrm{C}_{30} \mathrm{H}_{28} \mathrm{~N}_{4} \mathrm{VO}$ & 511.169712 & 511.169722 & 21 & 12.84 \\
$\mathrm{C}_{31} \mathrm{H}_{30} \mathrm{~N}_{4} \mathrm{VO}$ & 525.185379 & 525.185373 & -12 & 8.68 \\
$\mathrm{C}_{32} \mathrm{H}_{32} \mathrm{~N}_{4} \mathrm{VO}$ & 539.201018 & 539.201023 & 8 & 10.37 \\
$\mathrm{C}_{33} \mathrm{H}_{34} \mathrm{~N}_{4} \mathrm{VO}$ & 553.216665 & 553.216673 & 14 & 7.40 \\
$\mathrm{C}_{34} \mathrm{H}_{36} \mathrm{~N}_{4} \mathrm{VO}$ & 567.232334 & 567.232323 & -2 & 3.64 \\
$\mathrm{C}_{35} \mathrm{H}_{38} \mathrm{~N}_{4} \mathrm{VO}$ & 581.247979 & 581.247973 & -10 & 2.85 \\
$\mathrm{C}_{36} \mathrm{H}_{40} \mathrm{~N}_{4} \mathrm{VO}$ & 595.26363 & 595.263623 & -12 & 2.55 \\
$\mathrm{C}_{37} \mathrm{H}_{42} \mathrm{~N}_{4} \mathrm{VO}$ & 609.279283 & 609.279273 & -16 & 1.79 \\
$\mathrm{C}_{38} \mathrm{H}_{44} \mathrm{~N}_{4} \mathrm{VO}$ & 623.294944 & 623.294923 & -33 & 1.37 \\
$\mathrm{C}_{39} \mathrm{H}_{46} \mathrm{~N}_{4} \mathrm{VO}$ & 637.310603 & 637.310573 & -47 & 1.59 \\
$\mathrm{C}_{40} \mathrm{H}_{48} \mathrm{~N}_{4} \mathrm{VO}$ & 651.326259 & 651.326223 & -55 & 1.51 \\
$\mathrm{C}_{41} \mathrm{H}_{50} \mathrm{~N}_{4} \mathrm{VO}$ & 665.341893 & 665.341873 & -30 & 1.07 \\
$\mathrm{C}_{42} \mathrm{H}_{52} \mathrm{~N}_{4} \mathrm{VO}$ & 679.357534 & 679.357523 & -16 & 0.64 \\
$\mathrm{C}_{43} \mathrm{H}_{54} \mathrm{~N}_{4} \mathrm{VO}$ & 693.373248 & 693.373173 & -107 & 0.48 \\
$\mathrm{C}_{44} \mathrm{H}_{56} \mathrm{~N}_{4} \mathrm{VO}$ & 707.388874 & 707.388823 & -71 & 0.38 \\
$\mathrm{C}_{45} \mathrm{H}_{58} \mathrm{~N}_{4} \mathrm{VO}$ & 721.404532 & 721.404473 & -81 & 0.27 \\
$\mathrm{C}_{46} \mathrm{H}_{60} \mathrm{~N}_{4} \mathrm{VO}$ & 735.420182 & 735.420123 & -8 & 0.19 \\
$\mathrm{C}_{47} \mathrm{H}_{62} \mathrm{~N}_{4} \mathrm{VO}$ & 749.435788 & 749.435774 & -19 & 0.14 \\
$\mathrm{C}_{48} \mathrm{H}_{64} \mathrm{~N}_{4} \mathrm{VO}$ & 763.45157 & 763.451424 & -192 & 0.13 \\
\hline
\end{tabular}

\begin{tabular}{ccccc} 
KMD = 0.4145 & Vanadyl Porphyrins “DBE $=\mathbf{2 0 ”}$ & \multicolumn{2}{c}{ RMS = 49 ppb } & \\
\hline $\begin{array}{c}\text { Molecular } \\
\text { Formula }\end{array}$ & $\mathrm{m} / \mathbf{z}$ observed & $\begin{array}{c}\mathrm{m} / \mathbf{z} \\
\text { calculated }\end{array}$ & $\begin{array}{c}\text { Mass Error } \\
\text { ppb }\end{array}$ & $\begin{array}{c}\text { Relative } \\
\text { Abundance }\end{array}$ \\
\hline $\mathrm{C}_{25} \mathrm{H}_{16} \mathrm{~N}_{4} \mathrm{VO}$ & 439.075823 & 439.075822 & -1 & 0.93 \\
$\mathrm{C}_{26} \mathrm{H}_{18} \mathrm{~N}_{4} \mathrm{VO}$ & 453.091475 & 453.091472 & -5 & 3.16 \\
$\mathrm{C}_{27} \mathrm{H}_{20} \mathrm{~N}_{4} \mathrm{VO}$ & 467.107107 & 467.107122 & 33 & 6.81 \\
$\mathrm{C}_{28} \mathrm{H}_{22} \mathrm{~N}_{4} \mathrm{VO}$ & 481.122761 & 481.122772 & 23 & 8.85 \\
$\mathrm{C}_{29} \mathrm{H}_{24} \mathrm{~N}_{4} \mathrm{VO}$ & 495.138424 & 495.138422 & -4 & 11.97 \\
$\mathrm{C}_{30} \mathrm{H}_{26} \mathrm{~N}_{4} \mathrm{VO}$ & 509.154064 & 509.154072 & 17 & 19.26 \\
$\mathrm{C}_{31} \mathrm{H}_{28} \mathrm{~N}_{4} \mathrm{VO}$ & 523.169715 & 523.169722 & 14 & 20.73 \\
$\mathrm{C}_{32} \mathrm{H}_{30} \mathrm{~N}_{4} \mathrm{VO}$ & 537.185365 & 537.185373 & 15 & 22.89 \\
$\mathrm{C}_{33} \mathrm{H}_{32} \mathrm{~N}_{4} \mathrm{VO}$ & 551.201017 & 551.201023 & 10 & 15.82 \\
$\mathrm{C}_{34} \mathrm{H}_{34} \mathrm{~N}_{4} \mathrm{VO}$ & 565.216668 & 565.216673 & 9 & 9.74 \\
$\mathrm{C}_{35} \mathrm{H}_{36} \mathrm{~N}_{4} \mathrm{VO}$ & 579.232316 & 579.232323 & 11 & 7.60 \\
$\mathrm{C}_{36} \mathrm{H}_{38} \mathrm{~N}_{4} \mathrm{VO}$ & 593.247982 & 593.247973 & -16 & 5.90
\end{tabular}




\begin{tabular}{|c|c|c|c|c|}
\hline $\mathrm{C}_{37} \mathrm{H}_{40} \mathrm{~N}_{4} \mathrm{VO}$ & 607.263624 & 607.263623 & -2 & 3.42 \\
\hline $\mathrm{C}_{38} \mathrm{H}_{42} \mathrm{~N}_{4} \mathrm{VO}$ & 621.279281 & 621.279273 & -12 & 1.79 \\
\hline $\mathrm{C}_{39} \mathrm{H}_{44} \mathrm{~N}_{4} \mathrm{VO}$ & 635.29494 & 635.294923 & -27 & 1.73 \\
\hline $\mathrm{C}_{40} \mathrm{H}_{46} \mathrm{~N}_{4} \mathrm{VO}$ & 649.310589 & 649.310573 & -25 & 1.79 \\
\hline $\mathrm{C}_{41} \mathrm{H}_{48} \mathrm{~N}_{4} \mathrm{VO}$ & 663.326244 & 663.326223 & -31 & 1.18 \\
\hline $\mathrm{C}_{42} \mathrm{H}_{50} \mathrm{~N}_{4} \mathrm{VO}$ & 677.341906 & 677.341873 & -49 & 0.72 \\
\hline $\mathrm{C}_{43} \mathrm{H}_{52} \mathrm{~N}_{4} \mathrm{VO}$ & 691.357572 & 691.357523 & -70 & 0.47 \\
\hline $\mathrm{C}_{44} \mathrm{H}_{54} \mathrm{~N}_{4} \mathrm{VO}$ & 705.373185 & 705.373173 & -17 & 0.46 \\
\hline $\mathrm{C}_{45} \mathrm{H}_{56} \mathrm{~N}_{4} \mathrm{VO}$ & 719.388911 & 719.388823 & -122 & 0.35 \\
\hline $\mathrm{C}_{46} \mathrm{H}_{58} \mathrm{~N}_{4} \mathrm{VO}$ & 733.404564 & 733.404473 & -124 & 0.28 \\
\hline $\mathrm{C}_{47} \mathrm{H}_{60} \mathrm{~N}_{4} \mathrm{VO}$ & 747.420158 & 747.420123 & -47 & 0.13 \\
\hline $\mathrm{C}_{48} \mathrm{H}_{62} \mathrm{~N}_{4} \mathrm{VO}$ & 761.435859 & 761.435774 & -112 & 0.14 \\
\hline$K M D=0.42786$ & \multicolumn{3}{|c|}{ Vanadyl Porphyrins “DBE = 21” } & 5 ppb \\
\hline $\begin{array}{c}\text { Molecular } \\
\text { Formula } \\
\end{array}$ & $\begin{array}{c}m / z \\
\text { observed }\end{array}$ & $\begin{array}{c}\mathrm{m} / \mathrm{z} \\
\text { calculated }\end{array}$ & $\begin{array}{c}\text { Mass Error } \\
\text { ppb }\end{array}$ & $\begin{array}{c}\text { Relative } \\
\text { Abundance }\end{array}$ \\
\hline $\mathrm{C}_{25} \mathrm{H}_{14} \mathrm{~N}_{4} \mathrm{VO}$ & 437.060157 & 437.060172 & 35 & 0.19 \\
\hline $\mathrm{C}_{26} \mathrm{H}_{16} \mathrm{~N}_{4} \mathrm{VO}$ & 451.075837 & 451.075822 & -34 & 1.15 \\
\hline $\mathrm{C}_{27} \mathrm{H}_{18} \mathrm{~N}_{4} \mathrm{VO}$ & 465.091465 & 465.091472 & 16 & 2.57 \\
\hline $\mathrm{C}_{28} \mathrm{H}_{20} \mathrm{~N}_{4} \mathrm{VO}$ & 479.107124 & 479.107122 & -3 & 3.13 \\
\hline $\mathrm{C}_{29} \mathrm{H}_{22} \mathrm{~N}_{4} \mathrm{VO}$ & 493.122762 & 493.122772 & 20 & 4.40 \\
\hline $\mathrm{C}_{30} \mathrm{H}_{24} \mathrm{~N}_{4} \mathrm{VO}$ & 507.138414 & 507.138422 & 16 & 8.13 \\
\hline $\mathrm{C}_{31} \mathrm{H}_{26} \mathrm{~N}_{4} \mathrm{VO}$ & 521.154061 & 521.154072 & 21 & 12.28 \\
\hline $\mathrm{C}_{32} \mathrm{H}_{28} \mathrm{~N}_{4} \mathrm{VO}$ & 535.169709 & 535.169722 & 26 & 19.04 \\
\hline $\mathrm{C}_{33} \mathrm{H}_{30} \mathrm{~N}_{4} \mathrm{VO}$ & 549.185367 & 549.185373 & 11 & 16.61 \\
\hline $\mathrm{C}_{34} \mathrm{H}_{32} \mathrm{~N}_{4} \mathrm{VO}$ & 563.201028 & 563.201023 & -9 & 9.30 \\
\hline $\mathrm{C}_{35} \mathrm{H}_{34} \mathrm{~N}_{4} \mathrm{VO}$ & 577.216673 & 577.216673 & -1 & 7.39 \\
\hline $\mathrm{C}_{36} \mathrm{H}_{36} \mathrm{~N}_{4} \mathrm{VO}$ & 591.232315 & 591.232323 & 12 & 6.08 \\
\hline $\mathrm{C}_{37} \mathrm{H}_{38} \mathrm{~N}_{4} \mathrm{VO}$ & 605.247983 & 605.247973 & -17 & 3.57 \\
\hline $\mathrm{C}_{38} \mathrm{H}_{40} \mathrm{~N}_{4} \mathrm{VO}$ & 619.263621 & 619.263623 & 3 & 1.86 \\
\hline $\mathrm{C}_{39} \mathrm{H}_{42} \mathrm{~N}_{4} \mathrm{VO}$ & 633.279297 & 633.279273 & -38 & 1.60 \\
\hline $\mathrm{C}_{40} \mathrm{H}_{44} \mathrm{~N}_{4} \mathrm{VO}$ & 647.294935 & 647.294923 & -18 & 1.53 \\
\hline $\mathrm{C}_{41} \mathrm{H}_{46} \mathrm{~N}_{4} \mathrm{VO}$ & 661.310592 & 661.310573 & -28 & 1.10 \\
\hline $\mathrm{C}_{42} \mathrm{H}_{48} \mathrm{~N}_{4} \mathrm{VO}$ & 675.326255 & 675.326223 & -47 & 0.68 \\
\hline $\mathrm{C}_{43} \mathrm{H}_{50} \mathrm{~N}_{4} \mathrm{VO}$ & 689.341954 & 689.341873 & -117 & 0.49 \\
\hline $\mathrm{C}_{44} \mathrm{H}_{52} \mathrm{~N}_{4} \mathrm{VO}$ & 703.357564 & 703.357523 & -59 & 0.43 \\
\hline $\mathrm{C}_{45} \mathrm{H}_{54} \mathrm{~N}_{4} \mathrm{VO}$ & 717.373182 & 717.373173 & -12 & 0.29 \\
\hline $\mathrm{C}_{46} \mathrm{H}_{56} \mathrm{~N}_{4} \mathrm{VO}$ & 731.388868 & 731.388823 & -61 & 0.26 \\
\hline
\end{tabular}




\begin{tabular}{ccccc}
$\mathrm{C}_{47} \mathrm{H}_{58} \mathrm{~N}_{4} \mathrm{VO}$ & 745.40454 & 745.404473 & -9 & 0.14 \\
$\mathrm{C}_{48} \mathrm{H}_{60} \mathrm{~N}_{4} \mathrm{VO}$ & 759.420203 & 759.420123 & -104 & 0.14 \\
\hline
\end{tabular}

$\mathrm{KMD}=\mathbf{0 . 4 4 1 2 4}$ Vanadyl Porphyrins "DBE $=22$ " $\quad \mathrm{RMS}=47 \mathrm{ppb}$

\begin{tabular}{ccccc}
\hline Molecular & $\begin{array}{c}\mathrm{m} / \mathrm{z} \\
\text { Formula }\end{array}$ & $\begin{array}{c}\mathrm{m} / \mathrm{z} \\
\text { calculated }\end{array}$ & $\begin{array}{c}\text { Mass Error } \\
\text { ppb }\end{array}$ & $\begin{array}{c}\text { Relative } \\
\text { Abundance }\end{array}$ \\
\hline $\mathrm{C}_{26} \mathrm{H}_{14} \mathrm{~N}_{4} \mathrm{VO}$ & 449.060173 & 449.060172 & -2 & 0.33 \\
$\mathrm{C}_{27} \mathrm{H}_{16} \mathrm{~N}_{4} \mathrm{VO}$ & 463.075799 & 463.075822 & 49 & 0.75 \\
$\mathrm{C}_{28} \mathrm{H}_{18} \mathrm{~N}_{4} \mathrm{VO}$ & 477.09147 & 477.091472 & 4 & 1.28 \\
$\mathrm{C}_{29} \mathrm{H}_{20} \mathrm{~N}_{4} \mathrm{VO}$ & 491.107127 & 491.107122 & -10 & 2.14 \\
$\mathrm{C}_{30} \mathrm{H}_{22} \mathrm{~N}_{4} \mathrm{VO}$ & 505.122763 & 505.122772 & 18 & 3.49 \\
$\mathrm{C}_{31} \mathrm{H}_{24} \mathrm{~N}_{4} \mathrm{VO}$ & 519.138418 & 519.138422 & 8 & 3.38 \\
$\mathrm{C}_{32} \mathrm{H}_{26} \mathrm{~N}_{4} \mathrm{VO}$ & 533.154074 & 533.154072 & -3 & 3.43 \\
$\mathrm{C}_{33} \mathrm{H}_{28} \mathrm{~N}_{4} \mathrm{VO}$ & 547.169723 & 547.169722 & -1 & 3.59 \\
$\mathrm{C}_{34} \mathrm{H}_{30} \mathrm{~N}_{4} \mathrm{VO}$ & 561.185368 & 561.185373 & 7 & 3.49 \\
$\mathrm{C}_{35} \mathrm{H}_{32} \mathrm{~N}_{4} \mathrm{VO}$ & 575.201031 & 575.201023 & -14 & 2.95 \\
$\mathrm{C}_{36} \mathrm{H}_{34} \mathrm{~N}_{4} \mathrm{VO}$ & 589.216686 & 589.216673 & -23 & 3.00 \\
$\mathrm{C}_{37} \mathrm{H}_{36} \mathrm{~N}_{4} \mathrm{VO}$ & 603.232337 & 603.232323 & -23 & 2.02 \\
$\mathrm{C}_{38} \mathrm{H}_{38} \mathrm{~N}_{4} \mathrm{VO}$ & 617.247985 & 617.247973 & -19 & 1.15 \\
$\mathrm{C}_{39} \mathrm{H}_{40} \mathrm{~N}_{4} \mathrm{VO}$ & 631.263626 & 631.263623 & -6 & 1.05 \\
$\mathrm{C}_{40} \mathrm{H}_{42} \mathrm{~N}_{4} \mathrm{VO}$ & 645.27931 & 645.279273 & -58 & 1.11 \\
$\mathrm{C}_{41} \mathrm{H}_{44} \mathrm{~N}_{4} \mathrm{VO}$ & 659.294914 & 659.294923 & 13 & 0.90 \\
$\mathrm{C}_{42} \mathrm{H}_{46} \mathrm{~N}_{4} \mathrm{VO}$ & 673.310618 & 673.310573 & -67 & 0.52 \\
$\mathrm{C}_{43} \mathrm{H}_{48} \mathrm{~N}_{4} \mathrm{VO}$ & 687.326261 & 687.326223 & -55 & 0.38 \\
$\mathrm{C}_{44} \mathrm{H}_{50} \mathrm{~N}_{4} \mathrm{VO}$ & 701.341944 & 701.341873 & -101 & 0.35 \\
$\mathrm{C}_{45} \mathrm{H}_{52} \mathrm{~N}_{4} \mathrm{VO}$ & 715.357524 & 715.357523 & -1 & 0.27 \\
$\mathrm{C}_{46} \mathrm{H}_{54} \mathrm{~N}_{4} \mathrm{VO}$ & 729.373119 & 729.373173 & 75 & 0.20 \\
$\mathrm{C}_{47} \mathrm{H}_{56} \mathrm{~N}_{4} \mathrm{VO}$ & 743.388927 & 743.388823 & -140 & 0.12 \\
$\mathrm{C}_{48} \mathrm{H}_{58} \mathrm{~N}_{4} \mathrm{VO}$ & 757.404503 & 757.404473 & -40 & \\
& & & & \\
\hline
\end{tabular}

\begin{tabular}{ccccc} 
KMD = 0.45464 & Vanadyl Porphyrins “DBE = 23” & \multicolumn{2}{c}{ RMS = 60 ppb } \\
\hline Molecular & $\begin{array}{c}\mathrm{m} / \mathbf{z} \\
\text { observed }\end{array}$ & $\begin{array}{c}\mathrm{m} / \mathbf{z} \\
\text { calculated }\end{array}$ & $\begin{array}{c}\text { Mass Error } \\
\text { ppb }\end{array}$ & $\begin{array}{c}\text { Relative } \\
\text { Abundance }\end{array}$ \\
\hline Formula & 475.075802 & 475.075822 & 42 & 0.42 \\
$\mathrm{C}_{28} \mathrm{H}_{16} \mathrm{~N}_{4} \mathrm{VO}$ & 489.091474 & 489.091472 & -3 & 0.77 \\
$\mathrm{C}_{29} \mathrm{H}_{18} \mathrm{~N}_{4} \mathrm{VO}$ & 503.107123 & 503.107122 & -1 & 1.25 \\
$\mathrm{C}_{30} \mathrm{H}_{20} \mathrm{~N}_{4} \mathrm{VO}$ & 517.122765 & 517.122772 & 14 & 1.30 \\
$\mathrm{C}_{31} \mathrm{H}_{22} \mathrm{~N}_{4} \mathrm{VO}$ & 531.138417 & 531.138422 & 10 & 1.59 \\
$\mathrm{C}_{32} \mathrm{H}_{24} \mathrm{~N}_{4} \mathrm{VO}$ & &
\end{tabular}




\begin{tabular}{|c|c|c|c|c|}
\hline $\mathrm{C}_{33} \mathrm{H}_{26} \mathrm{~N}_{4} \mathrm{VO}$ & 545.154072 & 545.154072 & 1 & 2.39 \\
\hline $\mathrm{C}_{34} \mathrm{H}_{28} \mathrm{~N}_{4} \mathrm{VO}$ & 559.169723 & 559.169722 & -1 & 2.57 \\
\hline $\mathrm{C}_{35} \mathrm{H}_{30} \mathrm{~N}_{4} \mathrm{VO}$ & 573.185372 & 573.185373 & 1 & 2.91 \\
\hline $\mathrm{C}_{36} \mathrm{H}_{32} \mathrm{~N}_{4} \mathrm{VO}$ & 587.201025 & 587.201023 & -4 & 3.73 \\
\hline $\mathrm{C}_{37} \mathrm{H}_{34} \mathrm{~N}_{4} \mathrm{VO}$ & 601.216679 & 601.216673 & -10 & 3.00 \\
\hline $\mathrm{C}_{38} \mathrm{H}_{36} \mathrm{~N}_{4} \mathrm{VO}$ & 615.232325 & 615.232323 & -4 & 1.52 \\
\hline $\mathrm{C}_{39} \mathrm{H}_{38} \mathrm{~N}_{4} \mathrm{VO}$ & 629.248009 & 629.247973 & -58 & 1.10 \\
\hline $\mathrm{C}_{40} \mathrm{H}_{40} \mathrm{~N}_{4} \mathrm{VO}$ & 643.263639 & 643.263623 & -25 & 1.15 \\
\hline $\mathrm{C}_{41} \mathrm{H}_{42} \mathrm{~N}_{4} \mathrm{VO}$ & 657.279301 & 657.279273 & -43 & 0.84 \\
\hline $\mathrm{C}_{42} \mathrm{H}_{44} \mathrm{~N}_{4} \mathrm{VO}$ & 671.294996 & 671.294923 & -108 & 0.53 \\
\hline $\mathrm{C}_{43} \mathrm{H}_{46} \mathrm{~N}_{4} \mathrm{VO}$ & 685.310582 & 685.310573 & -14 & 0.42 \\
\hline $\mathrm{C}_{44} \mathrm{H}_{48} \mathrm{~N}_{4} \mathrm{VO}$ & 699.32629 & 699.326223 & -95 & 0.33 \\
\hline $\mathrm{C}_{45} \mathrm{H}_{50} \mathrm{~N}_{4} \mathrm{VO}$ & 713.341957 & 713.341873 & -118 & 0.27 \\
\hline $\mathrm{C}_{46} \mathrm{H}_{52} \mathrm{~N}_{4} \mathrm{VO}$ & 727.357529 & 727.357523 & -8 & 0.23 \\
\hline $\mathrm{C}_{47} \mathrm{H}_{54} \mathrm{~N}_{4} \mathrm{VO}$ & 741.373309 & 741.373173 & -83 & 0.17 \\
\hline $\mathrm{C}_{48} \mathrm{H}_{56} \mathrm{~N}_{4} \mathrm{VO}$ & 755.388838 & 755.388823 & -20 & 0.14 \\
\hline KMD = 0.46804 & Vanadyl Porphy & 1s "DBE = 24" & RMS $=5$ & \\
\hline $\begin{array}{c}\text { Molecular } \\
\text { Formula } \\
\end{array}$ & $\begin{array}{c}\mathrm{m} / \mathrm{z} \\
\text { observed }\end{array}$ & $\begin{array}{c}\mathrm{m} / \mathrm{z} \\
\text { calculated }\end{array}$ & $\begin{array}{l}\text { Mass Error } \\
\text { ppb }\end{array}$ & $\begin{array}{c}\text { Relative } \\
\text { Abundance }\end{array}$ \\
\hline $\mathrm{C}_{29} \mathrm{H}_{16} \mathrm{~N}_{4} \mathrm{VO}$ & 487.075793 & 487.075822 & 60 & 0.25 \\
\hline $\mathrm{C}_{30} \mathrm{H}_{18} \mathrm{~N}_{4} \mathrm{VO}$ & 501.0914721 & 501.0914951 & -45 & 0.38 \\
\hline $\mathrm{C}_{31} \mathrm{H}_{20} \mathrm{~N}_{4} \mathrm{VO}$ & 515.1071222 & 515.1071006 & 41 & 0.46 \\
\hline $\mathrm{C}_{32} \mathrm{H}_{22} \mathrm{~N}_{4} \mathrm{VO}$ & 529.1222772 & 515.1227911 & -35 & 0.52 \\
\hline $\mathrm{C}_{33} \mathrm{H}_{24} \mathrm{~N}_{4} \mathrm{VO}$ & 543.138408 & 543.138422 & 26 & 0.82 \\
\hline $\mathrm{C}_{34} \mathrm{H}_{26} \mathrm{~N}_{4} \mathrm{VO}$ & 557.154088 & 557.154072 & -29 & 0.81 \\
\hline $\mathrm{C}_{35} \mathrm{H}_{28} \mathrm{~N}_{4} \mathrm{VO}$ & 571.169723 & 571.169722 & 1 & 0.84 \\
\hline $\mathrm{C}_{36} \mathrm{H}_{30} \mathrm{~N}_{4} \mathrm{VO}$ & 585.185378 & 585.185373 & -9 & 1.29 \\
\hline $\mathrm{C}_{37} \mathrm{H}_{32} \mathrm{~N}_{4} \mathrm{VO}$ & 599.201028 & 599.201023 & -9 & 1.27 \\
\hline $\mathrm{C}_{38} \mathrm{H}_{34} \mathrm{~N}_{4} \mathrm{VO}$ & 613.216688 & 613.216673 & -25 & 0.91 \\
\hline $\mathrm{C}_{39} \mathrm{H}_{36} \mathrm{~N}_{4} \mathrm{VO}$ & 627.232333 & 627.232323 & -16 & 0.72 \\
\hline $\mathrm{C}_{40} \mathrm{H}_{38} \mathrm{~N}_{4} \mathrm{VO}$ & 641.24799 & 641.247973 & -26 & 0.86 \\
\hline $\mathrm{C}_{41} \mathrm{H}_{40} \mathrm{~N}_{4} \mathrm{VO}$ & 655.263629 & 655.263623 & -9 & 0.77 \\
\hline $\mathrm{C}_{42} \mathrm{H}_{42} \mathrm{~N}_{4} \mathrm{VO}$ & 669.27932 & 669.279273 & -71 & 0.48 \\
\hline $\mathrm{C}_{43} \mathrm{H}_{44} \mathrm{~N}_{4} \mathrm{VO}$ & 683.294963 & 683.294923 & -59 & 0.33 \\
\hline $\mathrm{C}_{44} \mathrm{H}_{46} \mathrm{~N}_{4} \mathrm{VO}$ & 697.310605 & 697.310573 & -45 & 0.24 \\
\hline $\mathrm{C}_{45} \mathrm{H}_{48} \mathrm{~N}_{4} \mathrm{VO}$ & 711.326289 & 711.326223 & -93 & 0.26 \\
\hline $\mathrm{C}_{46} \mathrm{H}_{50} \mathrm{~N}_{4} \mathrm{VO}$ & 725.341785 & 725.341873 & 122 & 0.18 \\
\hline
\end{tabular}




\begin{tabular}{ccccc}
$\mathrm{C}_{47} \mathrm{H}_{52} \mathrm{~N}_{4} \mathrm{VO}$ & 739.3575232 & 739.357472 & 69 & 0.12 \\
$\mathrm{C}_{48} \mathrm{H}_{54} \mathrm{~N}_{4} \mathrm{VO}$ & 753.373316 & 753.373173 & 89 & 0.10 \\
\hline KMD = 0.48144 & Vanadyl Porphyrins “DBE = 25” & RMS = 46 ppb \\
\hline Molecular & $\mathrm{m} / \mathrm{z}$ & $\mathrm{m} / \mathrm{z}$ & Mass Error & Relative \\
\hline Formula & observed & calculated & ppb & Abundance \\
\hline $\mathrm{C}_{34} \mathrm{H}_{24} \mathrm{~N}_{4} \mathrm{VO}$ & 555.138425 & 555.138422 & -3 & 0.34 \\
$\mathrm{C}_{35} \mathrm{H}_{26} \mathrm{~N}_{4} \mathrm{VO}$ & 569.154083 & 569.154072 & -10 & 0.38 \\
$\mathrm{C}_{36} \mathrm{H}_{28} \mathrm{~N}_{4} \mathrm{VO}$ & 583.169736 & 583.169722 & -14 & 0.44 \\
$\mathrm{C}_{37} \mathrm{H}_{30} \mathrm{~N}_{4} \mathrm{VO}$ & 597.185371 & 597.185373 & 2 & 0.58 \\
$\mathrm{C}_{38} \mathrm{H}_{32} \mathrm{~N}_{4} \mathrm{VO}$ & 611.201009 & 611.201023 & 13 & 0.38 \\
$\mathrm{C}_{39} \mathrm{H}_{34} \mathrm{~N}_{4} \mathrm{VO}$ & 625.216683 & 625.216673 & -10 & 0.33 \\
$\mathrm{C}_{40} \mathrm{H}_{36} \mathrm{~N}_{4} \mathrm{VO}$ & 639.23233 & 639.232323 & -7 & 0.50 \\
$\mathrm{C}_{41} \mathrm{H}_{48} \mathrm{~N}_{4} \mathrm{VO}$ & 653.248023 & 653.247973 & -51 & 0.45 \\
$\mathrm{C}_{42} \mathrm{H}_{40} \mathrm{~N}_{4} \mathrm{VO}$ & 667.263668 & 667.263623 & -45 & 0.39 \\
$\mathrm{C}_{43} \mathrm{H}_{42} \mathrm{~N}_{4} \mathrm{VO}$ & 681.279282 & 681.279273 & -9 & 0.29 \\
$\mathrm{C}_{44} \mathrm{H}_{44} \mathrm{~N}_{4} \mathrm{VO}$ & 695.294997 & 695.294923 & -74 & 0.24 \\
$\mathrm{C}_{45} \mathrm{H}_{46} \mathrm{~N}_{4} \mathrm{VO}$ & 709.310555 & 709.310573 & 18 & 0.21 \\
$\mathrm{C}_{46} \mathrm{H}_{48} \mathrm{~N}_{4} \mathrm{VO}$ & 723.326361 & 723.326223 & -138 & 0.18 \\
\hline
\end{tabular}

$\mathrm{KMD}=0.49482$ Vanadyl Porphyrins “DBE $=26$ " $\quad \mathrm{RMS}=66 \mathrm{ppb}$

\begin{tabular}{ccccc}
\hline $\begin{array}{c}\text { Molecular } \\
\text { Formula }\end{array}$ & $\begin{array}{c}\mathrm{m} / \mathrm{z} \\
\text { observed }\end{array}$ & $\begin{array}{c}\mathrm{m} / \mathrm{z} \\
\text { calculated }\end{array}$ & $\begin{array}{c}\text { Mass Error } \\
\text { ppb }\end{array}$ & $\begin{array}{c}\text { Relative } \\
\text { Abundance }\end{array}$ \\
\hline $\mathrm{C}_{34} \mathrm{H}_{22} \mathrm{~N}_{4} \mathrm{VO}$ & 553.122832 & 553.122772 & -108 & 0.14 \\
$\mathrm{C}_{35} \mathrm{H}_{24} \mathrm{~N}_{4} \mathrm{VO}$ & 567.138411 & 567.138422 & 19 & 0.18 \\
$\mathrm{C}_{36} \mathrm{H}_{26} \mathrm{~N}_{4} \mathrm{VO}$ & 581.154055 & 581.154072 & 31 & 0.22 \\
$\mathrm{C}_{37} \mathrm{H}_{28} \mathrm{~N}_{4} \mathrm{VO}$ & 595.16971 & 595.169722 & 21 & 0.32 \\
$\mathrm{C}_{38} \mathrm{H}_{30} \mathrm{~N}_{4} \mathrm{VO}$ & 609.185324 & 609.185373 & 8 & 0.32 \\
$\mathrm{C}_{39} \mathrm{H}_{32} \mathrm{~N}_{4} \mathrm{VO}$ & 623.2010226 & 623.2010564 & -58 & 0.35 \\
$\mathrm{C}_{40} \mathrm{H}_{34} \mathrm{~N}_{4} \mathrm{VO}$ & 637.21671 & 637.216673 & -24 & 0.35 \\
$\mathrm{C}_{41} \mathrm{H}_{36} \mathrm{~N}_{4} \mathrm{VO}$ & 651.232338 & 651.232323 & -4 & 0.41 \\
$\mathrm{C}_{42} \mathrm{H}_{38} \mathrm{~N}_{4} \mathrm{VO}$ & 665.247976 & 665.247973 & -54 & 0.36 \\
$\mathrm{C}_{43} \mathrm{H}_{40} \mathrm{~N}_{4} \mathrm{VO}$ & 679.263652 & 679.263623 & -42 & 0.21 \\
$\mathrm{C}_{44} \mathrm{H}_{42} \mathrm{~N}_{4} \mathrm{VO}$ & 693.279393 & 693.279273 & -173 & 0.20 \\
$\mathrm{C}_{45} \mathrm{H}_{44} \mathrm{~N}_{4} \mathrm{VO}$ & 707.294958 & 707.294923 & -49 & 0.19 \\
$\mathrm{C}_{46} \mathrm{H}_{46} \mathrm{~N}_{4} \mathrm{VO}$ & 721.310573 & 721.3105596 & 18 & 0.13 \\
$\mathrm{C}_{47} \mathrm{H}_{48} \mathrm{~N}_{4} \mathrm{VO}$ & 735.326243 & 735.326223 & -27 & 0.12 \\
\hline
\end{tabular}




\section{Table 2. Nickel Porphyrin distribution in fraction $E_{6}$}

\begin{tabular}{ccccc} 
KMD = 0.36772 & Nickel Porphyrins “DBE = 17” & \multicolumn{2}{c}{ RMS = 73 ppb } & \\
\hline Molecular & $\begin{array}{c}\mathrm{m} / \mathbf{z} \\
\text { observed }\end{array}$ & $\begin{array}{c}\mathrm{m} / \mathbf{z} \\
\text { calculated }\end{array}$ & $\begin{array}{c}\text { Mass Error } \\
\text { pp b }\end{array}$ & $\begin{array}{c}\text { Relative } \\
\text { Abundance }\end{array}$ \\
\hline $\mathrm{C}_{25} \mathrm{H}_{22} \mathrm{~N}_{4} \mathrm{Ni}$ & 436.119318 & 436.119241 & -177 & 1.04 \\
$\mathrm{C}_{26} \mathrm{H}_{24} \mathrm{~N}_{4} \mathrm{Ni}$ & 450.134911 & 450.134891 & -43 & 5.81 \\
$\mathrm{C}_{27} \mathrm{H}_{26} \mathrm{~N}_{4} \mathrm{Ni}$ & 464.150543 & 464.150541 & -4 & 18.20 \\
$\mathrm{C}_{28} \mathrm{H}_{28} \mathrm{~N}_{4} \mathrm{Ni}$ & 478.166184 & 478.166191 & 16 & 20.42 \\
$\mathrm{C}_{29} \mathrm{H}_{30} \mathrm{~N}_{4} \mathrm{Ni}$ & 492.181844 & 492.181841 & -5 & 17.05 \\
$\mathrm{C}_{30} \mathrm{H}_{32} \mathrm{~N}_{4} \mathrm{Ni}$ & 506.197496 & 506.197491 & -10 & 11.67 \\
$\mathrm{C}_{31} \mathrm{H}_{34} \mathrm{~N}_{4} \mathrm{Ni}$ & 520.213158 & 520.213141 & -33 & 5.36 \\
$\mathrm{C}_{32} \mathrm{H}_{36} \mathrm{~N}_{4} \mathrm{Ni}$ & 534.228799 & 534.228791 & -14 & 3.45 \\
$\mathrm{C}_{33} \mathrm{H}_{38} \mathrm{~N}_{4} \mathrm{Ni}$ & 548.244463 & 548.244442 & -4 & 1.98 \\
$\mathrm{C}_{34} \mathrm{H}_{40} \mathrm{~N}_{4} \mathrm{Ni}$ & 562.260131 & 562.260092 & -69 & 0.86 \\
$\mathrm{C}_{35} \mathrm{H}_{42} \mathrm{~N}_{4} \mathrm{Ni}$ & 576.275767 & 576.275742 & -44 & 0.54 \\
$\mathrm{C}_{36} \mathrm{H}_{44} \mathrm{~N}_{4} \mathrm{Ni}$ & 590.291402 & 590.291392 & -17 & 0.43 \\
$\mathrm{C}_{37} \mathrm{H}_{46} \mathrm{~N}_{4} \mathrm{Ni}$ & 604.307046 & 604.307042 & -7 & 0.15 \\
$\mathrm{C}_{38} \mathrm{H}_{48} \mathrm{~N}_{4} \mathrm{Ni}$ & 618.322557 & 618.322692 & 181 & 0.12
\end{tabular}

\begin{tabular}{ccccc} 
KMD = 0.36897 & Nickel Porphyrins “DBE = 18” & \multicolumn{2}{c}{ RMS = 102 ppb } \\
\hline Molecular & $\begin{array}{c}\mathrm{m} / \mathbf{z} \\
\text { observed }\end{array}$ & $\begin{array}{c}\mathrm{m} / \mathbf{z} \\
\text { calculated }\end{array}$ & $\begin{array}{c}\text { Mass Error } \\
\text { ppb }\end{array}$ & $\begin{array}{c}\text { Relative } \\
\text { Abundance }\end{array}$ \\
\hline Formula & 434.103717 & 434.103591 & -291 & 0.48 \\
$\mathrm{C}_{25} \mathrm{H}_{20} \mathrm{~N}_{4} \mathrm{Ni}$ & 448.119336 & 448.119241 & -211 & 1.87 \\
$\mathrm{C}_{26} \mathrm{H}_{22} \mathrm{~N}_{4} \mathrm{Ni}$ & 462.134932 & 462.134891 & -89 & 6.97 \\
$\mathrm{C}_{27} \mathrm{H}_{24} \mathrm{~N}_{4} \mathrm{Ni}$ & 476.150553 & 476.150541 & -24 & 11.69 \\
$\mathrm{C}_{28} \mathrm{H}_{26} \mathrm{~N}_{4} \mathrm{Ni}$ & 490.166198 & 490.166191 & -14 & 18.94 \\
$\mathrm{C}_{29} \mathrm{H}_{28} \mathrm{~N}_{4} \mathrm{Ni}$ & 504.181848 & 504.181841 & -14 & 25.29 \\
$\mathrm{C}_{30} \mathrm{H}_{30} \mathrm{~N}_{4} \mathrm{Ni}$ & 518.197493 & 518.197491 & -2 & 19.46 \\
$\mathrm{C}_{31} \mathrm{H}_{32} \mathrm{~N}_{4} \mathrm{Ni}$ & 532.213148 & 532.213141 & -13 & 12.71 \\
$\mathrm{C}_{32} \mathrm{H}_{34} \mathrm{~N} \mathrm{~N}_{4} \mathrm{Ni}$ & 546.228809 & 546.228791 & -31 & 4.23 \\
$\mathrm{C}_{33} \mathrm{H}_{36} \mathrm{~N}_{4} \mathrm{Ni}$ & 560.244462 & 560.244442 & -37 & 2.10 \\
$\mathrm{C}_{34} \mathrm{H}_{38} \mathrm{~N}_{4} \mathrm{Ni}$ & 574.260124 & 574.260092 & -57 & 1.18 \\
$\mathrm{C}_{35} \mathrm{H}_{40} \mathrm{~N}_{4} \mathrm{Ni}$ & 588.27576 & 588.275742 & -31 & 0.92 \\
$\mathrm{C}_{36} \mathrm{H}_{42} \mathrm{~N}_{4} \mathrm{Ni}$ & 602.291433 & 602.291392 & -68 & 0.46 \\
$\mathrm{C}_{37} \mathrm{H}_{44} \mathrm{~N}_{4} \mathrm{Ni}$ & 616.307002 & 616.307042 & 65 & 0.10 \\
$\mathrm{C}_{38} \mathrm{H}_{46} \mathrm{~N}_{4} \mathrm{Ni}$ & & & & \\
\hline
\end{tabular}




\begin{tabular}{ccccc} 
KMD = 0.39434 & Nickel Porphyrins “DBE = 19” & \multicolumn{2}{c}{ RMS = 163 ppb } \\
\hline Molecular & $\begin{array}{c}\mathrm{m} / \mathbf{z} \\
\text { observed }\end{array}$ & $\begin{array}{c}\mathrm{m} / \mathrm{z} \\
\text { calculated }\end{array}$ & $\begin{array}{c}\text { Mass Error } \\
\text { ppb }\end{array}$ & $\begin{array}{c}\text { Relative } \\
\text { Abundance }\end{array}$ \\
\hline Formula & 446.103654 & 446.103591 & -142 & 1.04 \\
$\mathrm{C}_{26} \mathrm{H}_{20} \mathrm{~N}_{4} \mathrm{Ni}$ & 460.119298 & 460.119241 & -124 & 2.68 \\
$\mathrm{C}_{27} \mathrm{H}_{22} \mathrm{~N} \mathrm{~N}_{4} \mathrm{Ni}$ & 474.13495 & 474.134891 & -123 & 2.51 \\
$\mathrm{C}_{28} \mathrm{H}_{24} \mathrm{~N}_{4} \mathrm{Ni}$ & 488.150617 & 488.150541 & -155 & 2.05 \\
$\mathrm{C}_{29} \mathrm{H}_{26} \mathrm{~N}_{4} \mathrm{Ni}$ & 502.166303 & 502.166191 & -223 & 1.68 \\
$\mathrm{C}_{30} \mathrm{H}_{28} \mathrm{~N}_{4} \mathrm{Ni}$ & 516.181941 & 516.181841 & -193 & 1.14 \\
$\mathrm{C}_{31} \mathrm{H}_{30} \mathrm{~N}_{4} \mathrm{Ni}$ & 530.197587 & 530.197491 & -18 & 1.12 \\
$\mathrm{C}_{32} \mathrm{H}_{32} \mathrm{~N}_{4} \mathrm{Ni}$ & 544.213208 & 544.213141 & -122 & 1.05 \\
$\mathrm{C}_{33} \mathrm{H}_{34} \mathrm{~N}_{4} \mathrm{Ni}$ & 558.22888 & 558.228791 & -159 & 0.50 \\
$\mathrm{C}_{34} \mathrm{H}_{36} \mathrm{~N}_{4} \mathrm{Ni}$ & 572.244508 & 572.244442 & -115 & 0.30 \\
$\mathrm{C}_{35} \mathrm{H}_{38} \mathrm{~N}_{4} \mathrm{Ni}$ & 586.259585 & 586.260092 & 164 & 0.33 \\
$\mathrm{C}_{36} \mathrm{H}_{40} \mathrm{~N}_{4} \mathrm{Ni}$ & 600.275462 & 600.275742 & 180 & 0.22 \\
$\mathrm{C}_{37} \mathrm{H}_{42} \mathrm{~N}_{4} \mathrm{Ni}$ & 614.290921 & 614.291092 & 267 & 0.15 \\
$\mathrm{C}_{38} \mathrm{H}_{44} \mathrm{~N}_{4} \mathrm{Ni}$ & & &
\end{tabular}

\begin{tabular}{ccccc} 
KMD = 0.40774 & Nickel Porphyrins “DBE = 20” & \multicolumn{2}{c}{ RMS = 131 ppb } & \\
\hline Molecular & $\begin{array}{c}\mathrm{m} / \mathbf{z} \\
\text { observed }\end{array}$ & $\begin{array}{c}\mathrm{m} / \mathbf{z} \\
\text { calculated }\end{array}$ & $\begin{array}{c}\text { Mass Error } \\
\text { ppb }\end{array}$ & $\begin{array}{c}\text { Relative } \\
\text { Abundance }\end{array}$ \\
\hline Formula & 444.088015 & 444.087941 & -167 & 0.35 \\
$\mathrm{C}_{26} \mathrm{H}_{18} \mathrm{~N}_{4} \mathrm{Ni}$ & 458.103694 & 458.103591 & -224 & 1.08 \\
$\mathrm{C}_{27} \mathrm{H}_{20} \mathrm{~N}_{4} \mathrm{Ni}$ & 472.119349 & 472.119241 & -228 & 1.35 \\
$\mathrm{C}_{28} \mathrm{H}_{22} \mathrm{~N}_{4} \mathrm{Ni}$ & 486.134965 & 486.134891 & -152 & 2.27 \\
$\mathrm{C}_{29} \mathrm{H}_{24} \mathrm{~N}_{4} \mathrm{Ni}$ & 500.150586 & 500.150541 & -90 & 4.94 \\
$\mathrm{C}_{30} \mathrm{H}_{26} \mathrm{~N}_{4} \mathrm{Ni}$ & 514.166228 & 514.166191 & -72 & 5.19 \\
$\mathrm{C}_{31} \mathrm{H}_{28} \mathrm{~N}_{4} \mathrm{Ni}$ & 528.181891 & 528.181841 & -95 & 3.57 \\
$\mathrm{C}_{32} \mathrm{H}_{30} \mathrm{~N}_{4} \mathrm{Ni}$ & 542.197537 & 542.197491 & -84 & 2.72 \\
$\mathrm{C}_{33} \mathrm{H}_{32} \mathrm{~N}_{4} \mathrm{Ni}$ & 556.213203 & 556.213141 & -111 & 1.29 \\
$\mathrm{C}_{34} \mathrm{H}_{34} \mathrm{~N}_{4} \mathrm{Ni}$ & 570.22884 & 570.228791 & -85 & 0.73 \\
$\mathrm{C}_{35} \mathrm{H}_{36} \mathrm{~N}_{4} \mathrm{Ni}$ & 584.244476 & 584.244442 & -59 & 0.62 \\
$\mathrm{C}_{36} \mathrm{H}_{38} \mathrm{~N}_{4} \mathrm{Ni}$ & 598.260136 & 598.260092 & -75 & 0.39 \\
$\mathrm{C}_{37} \mathrm{H}_{40} \mathrm{~N}_{4} \mathrm{Ni}$ & 612.275564 & 612.275742 & 116 & 0.11 \\
$\mathrm{C}_{38} \mathrm{H}_{42} \mathrm{~N}_{4} \mathrm{Ni}$ & & & &
\end{tabular}

\begin{tabular}{ccccc} 
KMD = 0.42130 & Nickel Porphyrins “DBE = 21” & \multicolumn{2}{c}{ RMS = 210 ppb } \\
\hline Molecular & $\begin{array}{c}\mathrm{m} / \mathbf{z} \\
\text { observed }\end{array}$ & $\begin{array}{c}\mathrm{m} / \mathbf{z} \\
\text { calculated }\end{array}$ & $\begin{array}{c}\text { Mass Error } \\
\text { ppb }\end{array}$ & $\begin{array}{c}\text { Relative } \\
\text { Abundance }\end{array}$ \\
\hline Formula & 456.088045 & 456.087941 & -228 & 1.08 \\
$\mathrm{C}_{27} \mathrm{H}_{20} \mathrm{~N}_{4} \mathrm{Ni}$ & 470.103735 & 470.103591 & -307 & 1.35 \\
$\mathrm{C}_{28} \mathrm{H}_{22} \mathrm{~N}_{4} \mathrm{Ni}$ & & & &
\end{tabular}




\begin{tabular}{lllll}
$\mathrm{C}_{29} \mathrm{H}_{24} \mathrm{~N} \mathrm{~N}_{4} \mathrm{Ni}$ & 484.119398 & 484.119241 & -324 & 2.27 \\
$\mathrm{C}_{30} \mathrm{H}_{26} \mathrm{~N}_{4} \mathrm{Ni}$ & 498.135027 & 498.134891 & -273 & 4.94 \\
$\mathrm{C}_{31} \mathrm{H}_{28} \mathrm{~N}_{4} \mathrm{Ni}$ & 512.150651 & 512.150541 & -214 & 5.19 \\
$\mathrm{C}_{32} \mathrm{H}_{30} \mathrm{~N} \mathrm{~N}_{4} \mathrm{Ni}$ & 526.166291 & 526.166191 & -189 & 3.57 \\
$\mathrm{C}_{33} \mathrm{H}_{32} \mathrm{~N}_{4} \mathrm{Ni}$ & 540.181921 & 540.181841 & -148 & 2.72 \\
$\mathrm{C}_{34} \mathrm{H}_{34} \mathrm{~N}_{4} \mathrm{Ni}$ & 554.197572 & 554.197491 & -146 & 1.29 \\
$\mathrm{C}_{35} \mathrm{H}_{36} \mathrm{~N}_{4} \mathrm{Ni}$ & 568.213232 & 568.213141 & -160 & 0.73 \\
$\mathrm{C}_{36} \mathrm{H}_{38} \mathrm{~N}_{4} \mathrm{Ni}$ & 582.228838 & 582.228791 & -81 & 0.62 \\
$\mathrm{C}_{37} \mathrm{H}_{40} \mathrm{~N}_{4} \mathrm{Ni}$ & 596.244033 & 596.244442 & -154 & 0.39 \\
$\mathrm{C}_{38} \mathrm{H}_{42} \mathrm{~N}_{4} \mathrm{Ni}$ & 610.259849 & 610.260092 & -161 & 0.11 \\
\hline
\end{tabular}

\begin{tabular}{ccccc} 
KMD = 0.43190 & Nickel Porphyrins “DBE = 22” & \multicolumn{2}{c}{ RMS = 358 ppb } & \\
\hline Molecular & $\begin{array}{c}\mathrm{m} / \mathbf{z} \\
\text { observed }\end{array}$ & $\begin{array}{c}\mathrm{m} / \mathbf{z} \\
\text { calculated }\end{array}$ & $\begin{array}{c}\text { Mass Error } \\
\text { ppb }\end{array}$ & $\begin{array}{c}\text { Relative } \\
\text { Abundance }\end{array}$ \\
\hline Formula & 482.10335 & 482.103591 & 490 & 0.74 \\
$\mathrm{C}_{29} \mathrm{H}_{20} \mathrm{~N}_{4} \mathrm{Ni}$ & 496.119105 & 496.119241 & 274 & 0.25 \\
$\mathrm{C}_{30} \mathrm{H}_{22} \mathrm{~N}_{4} \mathrm{Ni}$ & 510.135084 & 510.134891 & -378 & 0.26 \\
$\mathrm{C}_{31} \mathrm{H}_{24} \mathrm{~N}_{4} \mathrm{Ni}$ & 524.150689 & 524.150541 & -282 & 0.55 \\
$\mathrm{C}_{32} \mathrm{H}_{26} \mathrm{~N}_{4} \mathrm{Ni}$ & 538.166405 & 538.166191 & -397 & 1.09 \\
$\mathrm{C}_{33} \mathrm{H}_{28} \mathrm{~N}_{4} \mathrm{Ni}$ & 552.181975 & 552.181841 & -242 & 0.67 \\
$\mathrm{C}_{34} \mathrm{H}_{30} \mathrm{~N}_{4} \mathrm{Ni}$ & 566.197699 & 566.197491 & -367 & 0.38 \\
$\mathrm{C}_{35} \mathrm{H}_{32} \mathrm{~N}_{4} \mathrm{Ni}$ & 580.213416 & 580.213141 & -473 & 0.37 \\
$\mathrm{C}_{36} \mathrm{H}_{34} \mathrm{~N}_{4} \mathrm{Ni}$ & 594.228912 & 594.228791 & -203 & 0.29 \\
$\mathrm{C}_{37} \mathrm{H}_{36} \mathrm{~N}_{4} \mathrm{Ni}$ & 608.244652 & 608.244442 & -345 & 0.14 \\
$\mathrm{C}_{38} \mathrm{H}_{38} \mathrm{~N}_{4} \mathrm{Ni}$ & & & &
\end{tabular}

\begin{tabular}{ccccc} 
KMD = 0.44855 & Nickel Porphyrins “DBE = 23” & \multicolumn{2}{c}{ RMS = 365 ppb } \\
\hline Molecular & $\begin{array}{c}\mathrm{m} / \mathbf{z} \\
\text { observed }\end{array}$ & $\begin{array}{c}\mathrm{m} / \mathbf{z} \\
\text { calculated }\end{array}$ & $\begin{array}{c}\text { Mass Error } \\
\text { ppb }\end{array}$ & $\begin{array}{c}\text { Relative } \\
\text { Abundance }\end{array}$ \\
\hline Formula & 494.103717 & 494.103591 & -255 & 0.25 \\
$\mathrm{C}_{30} \mathrm{H}_{20} \mathrm{~N}_{4} \mathrm{Ni}$ & 508.119413 & 508.119241 & -338 & 0.33 \\
$\mathrm{C}_{31} \mathrm{H}_{22} \mathrm{~N}_{4} \mathrm{Ni}$ & 522.135042 & 522.134891 & -289 & 0.46 \\
$\mathrm{C}_{32} \mathrm{H}_{24} \mathrm{~N}_{4} \mathrm{Ni}$ & 536.150733 & 536.150541 & -358 & 0.56 \\
$\mathrm{C}_{33} \mathrm{H}_{26} \mathrm{~N}_{4} \mathrm{Ni}$ & 550.166481 & 550.166191 & -527 & 0.80 \\
$\mathrm{C}_{34} \mathrm{H}_{28} \mathrm{~N}_{4} \mathrm{Ni}$ & & &
\end{tabular}




\begin{tabular}{lllll}
$\mathrm{C}_{35} \mathrm{H}_{30} \mathrm{~N}_{4} \mathrm{Ni}$ & 564.181612 & 564.181841 & 405 & 0.50 \\
$\mathrm{C}_{36} \mathrm{H}_{32} \mathrm{~N}{ }_{4} \mathrm{Ni}$ & 578.197665 & 578.197491 & -300 & 0.42 \\
$\mathrm{C}_{37} \mathrm{H}_{34} \mathrm{~N}_{4} \mathrm{Ni}$ & 592.213364 & 592.213141 & -376 & 0.21 \\
\hline
\end{tabular}

\section{Table S3. $\mathrm{VO}_{2}$ porphyrin distribution in fraction $\mathrm{E}_{6}$}

\begin{tabular}{ccccc} 
KMD = 0.41062 & VO $_{\mathbf{2}}$ Porphyrins “DBE = 18” & RMS = 70 ppb & \\
\hline Molecular & $\begin{array}{c}\mathrm{m} / \mathbf{z} \\
\text { observed }\end{array}$ & $\begin{array}{c}\mathrm{m} / \mathbf{z} \\
\text { calculated }\end{array}$ & $\begin{array}{c}\text { Mass Error } \\
\text { ppb }\end{array}$ & $\begin{array}{c}\text { Relative } \\
\text { Abundance }\end{array}$ \\
\hline Formula & 487.133297 & 487.133337 & 82 & 2.17 \\
$\mathrm{C}_{27} \mathrm{H}_{24} \mathrm{~N}_{4} \mathrm{VO}_{2}$ & 501.148969 & 501.148987 & 36 & 3.45 \\
$\mathrm{C}_{28} \mathrm{H}_{26} \mathrm{~N}_{4} \mathrm{VO}_{2}$ & 515.164653 & 515.164637 & -31 & 4.24 \\
$\mathrm{C}_{29} \mathrm{H}_{28} \mathrm{~N}_{4} \mathrm{VO}_{2}$ & 529.180261 & 529.180287 & 49 & 5.97 \\
$\mathrm{C}_{30} \mathrm{H}_{30} \mathrm{~N}_{4} \mathrm{VO}_{2}$ & 543.195976 & 543.195937 & -71 & 3.56 \\
$\mathrm{C}_{31} \mathrm{H}_{32} \mathrm{~N}_{4} \mathrm{VO}_{2}$ & 557.211575 & 557.211587 & 22 & 2.11 \\
$\mathrm{C}_{32} \mathrm{H}_{34} \mathrm{~N}_{4} \mathrm{VO}_{2}$ & 571.227262 & 571.227237 & -43 & 1.28 \\
$\mathrm{C}_{33} \mathrm{H}_{36} \mathrm{~N}_{4} \mathrm{VO}_{2}$ & 585.242927 & 585.242887 & -68 & 0.70 \\
$\mathrm{C}_{34} \mathrm{H}_{38} \mathrm{~N}_{4} \mathrm{VO}_{2}$ & 599.258624 & 599.258537 & -144 & 0.32 \\
$\mathrm{C}_{35} \mathrm{H}_{40} \mathrm{~N}_{4} \mathrm{VO}_{2}$ & & &
\end{tabular}

\begin{tabular}{ccccc} 
KMD = 0.42404 & VO & Porphyrins “DBE = 19” & RMS = 118 ppb & \\
\hline Molecular & $\begin{array}{c}\mathrm{m} / \mathbf{z} \\
\text { observed }\end{array}$ & $\begin{array}{c}\mathrm{m} / \mathbf{z} \\
\text { calculated }\end{array}$ & $\begin{array}{c}\text { Mass Error } \\
\text { ppb }\end{array}$ & $\begin{array}{c}\text { Relative } \\
\text { Abundance }\end{array}$ \\
\hline Formula & 499.133341 & 499.133337 & -8 & 2.07 \\
$\mathrm{C}_{28} \mathrm{H}_{24} \mathrm{~N}_{4} \mathrm{VO}_{2}$ & 513.149021 & 513.148987 & -67 & 2.47 \\
$\mathrm{C}_{29} \mathrm{H}_{26} \mathrm{~N}_{4} \mathrm{VO}_{2}$ & 527.164615 & 527.164637 & 41 & 2.97 \\
$\mathrm{C}_{30} \mathrm{H}_{28} \mathrm{~N}_{4} \mathrm{VO}_{2}$ & 541.180246 & 541.180287 & 76 & 3.47 \\
$\mathrm{C}_{31} \mathrm{H}_{30} \mathrm{~N}_{4} \mathrm{VO}_{2}$ & 555.195923 & 555.195937 & 25 & 1.32 \\
$\mathrm{C}_{32} \mathrm{H}_{32} \mathrm{~N}_{4} \mathrm{VO}_{2}$ & 569.211535 & 569.211587 & 93 & 1.07 \\
$\mathrm{C}_{33} \mathrm{H}_{34} \mathrm{~N}_{4} \mathrm{VO}_{2}$ & 583.227238 & 583.227237 & -1 & 0.69 \\
$\mathrm{C}_{34} \mathrm{H}_{36} \mathrm{~N}_{4} \mathrm{VO}_{2}$ & 597.243066 & 597.242887 & -299 & 0.58 \\
$\mathrm{C}_{35} \mathrm{H}_{38} \mathrm{~N}_{4} \mathrm{VO}_{2}$ & 611.258613 & 611.258537 & -124 & 0.40 \\
$\mathrm{C}_{36} \mathrm{H}_{40} \mathrm{~N}_{4} \mathrm{VO}_{2}$ & & & &
\end{tabular}

\begin{tabular}{ccccc} 
KMD = 0.43174 & VO $_{\mathbf{2}}$ Porphyrins “DBE = 20” & RMS = 70 ppb & \\
\hline Molecular & $\begin{array}{c}\mathrm{m} / \mathbf{z} \\
\text { observed }\end{array}$ & $\begin{array}{c}\mathrm{m} / \mathbf{z} \\
\text { calculated }\end{array}$ & $\begin{array}{c}\text { Mass Error } \\
\text { ppb }\end{array}$ & $\begin{array}{c}\text { Relative } \\
\text { Abundance }\end{array}$ \\
\hline Formula & 483.102051 & 483.102037 & -28 & 1.14 \\
$\mathrm{C}_{27} \mathrm{H}_{20} \mathrm{~N}_{4} \mathrm{VO}_{2}$ & 497.117685 & 497.117687 & 3 & 1.78 \\
$\mathrm{C}_{28} \mathrm{H}_{22} \mathrm{~N}_{4} \mathrm{VO}_{2}$ & 511.133299 & 511.133337 & 75 & 1.71 \\
$\mathrm{C}_{29} \mathrm{H}_{24} \mathrm{~N}_{4} \mathrm{VO}_{2}$ & & & &
\end{tabular}




\begin{tabular}{|c|c|c|c|c|}
\hline $\mathrm{C}_{30} \mathrm{H}_{26} \mathrm{~N}_{4} \mathrm{VO}_{2}$ & 525.14898 & 525.148987 & 13 & 1.56 \\
\hline $\mathrm{C}_{31} \mathrm{H}_{28} \mathrm{~N}_{4} \mathrm{VO}_{2}$ & 539.164613 & 539.164637 & 45 & 1.26 \\
\hline $\mathrm{C}_{32} \mathrm{H}_{30} \mathrm{~N}_{4} \mathrm{VO}_{2}$ & 553.180273 & 553.180287 & 26 & 0.68 \\
\hline $\mathrm{C}_{33} \mathrm{H}_{32} \mathrm{~N}_{4} \mathrm{VO}_{2}$ & 567.195924 & 567.195937 & 24 & 0.61 \\
\hline $\mathrm{C}_{34} \mathrm{H}_{34} \mathrm{~N}_{4} \mathrm{VO}_{2}$ & 581.211486 & 581.211587 & 174 & 0.44 \\
\hline $\mathrm{C}_{35} \mathrm{H}_{36} \mathrm{~N}_{4} \mathrm{VO}_{2}$ & 595.227171 & 595.227237 & 112 & 0.41 \\
\hline $\mathrm{C}_{36} \mathrm{H}_{38} \mathrm{~N}_{4} \mathrm{VO}_{2}$ & 609.242813 & 609.242887 & 123 & 0.24 \\
\hline $\mathrm{KMD}=0.44514$ & $\mathrm{VO}_{2}$ Porphyrins “ & 'DBE = 21”' & RMS = 83 ppb & \\
\hline $\begin{array}{c}\text { Molecular } \\
\text { Formula } \\
\end{array}$ & $\begin{array}{c}m / z \\
\text { observed }\end{array}$ & $\begin{array}{c}m / z \\
\text { calculated }\end{array}$ & $\begin{array}{c}\text { Mass Error } \\
\text { ppb }\end{array}$ & $\begin{array}{c}\text { Relative } \\
\text { Abundance }\end{array}$ \\
\hline $\mathrm{C}_{27} \mathrm{H}_{18} \mathrm{~N}_{4} \mathrm{VO}_{2}$ & 481.086326 & 481.086387 & 126 & 0.58 \\
\hline $\mathrm{C}_{28} \mathrm{H}_{20} \mathrm{~N}_{4} \mathrm{VO}_{2}$ & 495.102082 & 495.102037 & -92 & 0.76 \\
\hline $\mathrm{C}_{29} \mathrm{H}_{22} \mathrm{~N}_{4} \mathrm{VO}_{2}$ & 509.117703 & 509.117687 & -32 & 0.82 \\
\hline $\mathrm{C}_{30} \mathrm{H}_{24} \mathrm{~N}_{4} \mathrm{VO}_{2}$ & 523.133355 & 523.133337 & -35 & 0.77 \\
\hline $\mathrm{C}_{31} \mathrm{H}_{26} \mathrm{~N}_{4} \mathrm{VO}_{2}$ & 537.148915 & 537.148987 & 135 & 1.05 \\
\hline $\mathrm{C}_{32} \mathrm{H}_{28} \mathrm{~N}_{4} \mathrm{VO}_{2}$ & 551.164595 & 551.164637 & 77 & 0.88 \\
\hline $\mathrm{C}_{33} \mathrm{H}_{30} \mathrm{~N}_{4} \mathrm{VO}_{2}$ & 565.180262 & 565.180287 & 45 & 0.61 \\
\hline $\mathrm{C}_{34} \mathrm{H}_{32} \mathrm{~N}_{4} \mathrm{VO}_{2}$ & 579.19588 & 579.195937 & 98 & 0.60 \\
\hline $\mathrm{C}_{35} \mathrm{H}_{34} \mathrm{~N}_{4} \mathrm{VO}_{2}$ & 593.211572 & 593.211587 & 25 & 0.36 \\
\hline $\mathrm{C}_{36} \mathrm{H}_{36} \mathrm{~N}_{4} \mathrm{VO}_{2}$ & 607.227202 & 607.227237 & 58 & 0.15 \\
\hline KMD = 0.45854 & $\mathrm{VO}_{2}$ Porphyrins " & 'DBE = 22” & RMS = 93 ppb & \\
\hline $\begin{array}{c}\text { Molecular } \\
\text { Formula } \\
\end{array}$ & $\begin{array}{c}\mathrm{m} / \mathrm{z} \\
\text { observed }\end{array}$ & $\begin{array}{c}\mathrm{m} / \mathrm{z} \\
\text { calculated }\end{array}$ & $\begin{array}{c}\text { Mass Error } \\
\text { ppb }\end{array}$ & $\begin{array}{c}\text { Relative } \\
\text { Abundance }\end{array}$ \\
\hline $\mathrm{C}_{30} \mathrm{H}_{22} \mathrm{~N}_{4} \mathrm{VO}_{2}$ & 521.117634 & 521.117687 & 101 & 0.39 \\
\hline $\mathrm{C}_{31} \mathrm{H}_{24} \mathrm{~N}_{4} \mathrm{VO}_{2}$ & 535.133266 & 535.133337 & 133 & 0.52 \\
\hline $\mathrm{C}_{32} \mathrm{H}_{26} \mathrm{~N}_{4} \mathrm{VO}_{2}$ & 549.148998 & 549.148987 & -19 & 0.21 \\
\hline $\mathrm{C}_{33} \mathrm{H}_{28} \mathrm{~N}_{4} \mathrm{VO}_{2}$ & 563.164711 & 563.164637 & -131 & 0.19 \\
\hline $\mathrm{C}_{34} \mathrm{H}_{30} \mathrm{~N}_{4} \mathrm{VO}_{2}$ & 577.180299 & 577.180287 & -21 & 0.21 \\
\hline $\mathrm{C}_{35} \mathrm{H}_{32} \mathrm{~N}_{4} \mathrm{VO}_{2}$ & 591.195926 & 591.195937 & 19 & 0.17 \\
\hline $\mathrm{C}_{36} \mathrm{H}_{34} \mathrm{~N}_{4} \mathrm{VO}_{2}$ & 605.211676 & 605.211587 & -147 & 0.11 \\
\hline
\end{tabular}

Table S4. $\mathrm{VO}_{3}$ porphyrin distribution in fraction $\mathrm{E}_{6}$

\begin{tabular}{ccccc} 
KMD = $\mathbf{0 . 4 3 3 5 4}$ & $\mathbf{V O}_{\mathbf{3}}$ Porphyrins “DBE = 18” & \multicolumn{2}{c}{ RMS = 37 ppb } & \\
\hline Molecular & $\begin{array}{c}\mathrm{m} / \mathbf{z} \\
\text { observed }\end{array}$ & $\begin{array}{c}\mathrm{m} / \mathbf{z} \\
\text { calculated }\end{array}$ & $\begin{array}{c}\text { Mass Error } \\
\text { ppb }\end{array}$ & $\begin{array}{c}\text { Relative } \\
\text { Abundance }\end{array}$ \\
\hline Formula & 531.159529 & 531.159552 & 43 & 0.19 \\
\hline $\mathrm{C}_{29} \mathrm{H}_{28} \mathrm{~N}_{4} \mathrm{VO}_{3}$ & 545.175177 & 545.175202 & 45 & 0.13 \\
$\mathrm{C}_{30} \mathrm{H}_{30} \mathrm{~N}_{4} \mathrm{VO}_{3}$ & & &
\end{tabular}




\begin{tabular}{lcccc}
$\mathrm{C}_{31} \mathrm{H}_{32} \mathrm{~N}_{4} \mathrm{VO}_{3}$ & 559.190811 & 559.190852 & 73 & 0.22 \\
$\mathrm{C}_{32} \mathrm{H}_{34} \mathrm{~N}_{4} \mathrm{VO}_{3}$ & 573.206493 & 573.206502 & 16 & 0.48 \\
$\mathrm{C}_{33} \mathrm{H}_{36} \mathrm{~N}_{4} \mathrm{VO}_{3}$ & 587.22217 & 587.222152 & -31 & 0.42 \\
$\mathrm{C}_{34} \mathrm{H}_{38} \mathrm{~N}_{4} \mathrm{VO}_{3}$ & 601.23779 & 601.237802 & 19 & 0.21 \\
\hline
\end{tabular}

\begin{tabular}{ccccc} 
KMD = 0.44694 & VO $_{3}$ Porphyrins “DBE = 19” & RMS = 67 ppb & \\
\hline Molecular & $\begin{array}{c}\mathrm{m} / \mathbf{z} \\
\text { observed }\end{array}$ & $\begin{array}{c}\mathrm{m} / \mathbf{z} \\
\text { calculated }\end{array}$ & $\begin{array}{c}\text { Mass Error } \\
\text { ppb }\end{array}$ & $\begin{array}{c}\text { Relative } \\
\text { Abundance }\end{array}$ \\
\hline Formula & 529.143907 & 529.143902 & -11 & 0.18 \\
$\mathrm{C}_{29} \mathrm{H}_{26} \mathrm{~N}_{4} \mathrm{VO}_{3}$ & 543.159499 & 543.159552 & 97 & 0.21 \\
$\mathrm{C}_{30} \mathrm{H}_{28} \mathrm{~N}_{4} \mathrm{VO}_{3}$ & 557.17522 & 557.175202 & -33 & 0.32 \\
$\mathrm{C}_{31} \mathrm{H}_{30} \mathrm{~N}_{4} \mathrm{VO}_{3}$ & 571.190803 & 571.190852 & 86 & 0.95 \\
$\mathrm{C}_{32} \mathrm{H}_{32} \mathrm{~N}_{4} \mathrm{VO}_{3}$ & 585.206551 & 585.206502 & -85 & 1.25 \\
$\mathrm{C}_{33} \mathrm{H}_{34} \mathrm{~N}_{4} \mathrm{VO}_{3}$ & 599.222136 & 599.222152 & 26 & 0.63 \\
$\mathrm{C}_{34} \mathrm{H}_{36} \mathrm{~N}_{4} \mathrm{VO}_{3}$ & 613.237855 & 613.237802 & -87 & 0.46 \\
$\mathrm{C}_{35} \mathrm{H}_{38} \mathrm{~N}_{4} \mathrm{VO}_{3}$ & 627.25342 & 627.253452 & 50 & 0.20 \\
$\mathrm{C}_{36} \mathrm{H}_{40} \mathrm{~N}_{4} \mathrm{VO}_{3}$ & & & &
\end{tabular}

\begin{tabular}{ccccc} 
KMD = 0.46033 & VO $_{3}$ Porphyrins “DBE = 20” & RMS = 65 ppb & \\
\hline Molecular & $\begin{array}{c}\mathrm{m} / \mathbf{z} \\
\text { observed }\end{array}$ & $\begin{array}{c}\mathrm{m} / \mathbf{z} \\
\text { calculated }\end{array}$ & $\begin{array}{c}\text { Mass Error } \\
\text { ppb }\end{array}$ & $\begin{array}{c}\text { Relative } \\
\text { Abundance }\end{array}$ \\
\hline $\mathrm{C}_{30} \mathrm{H}_{26} \mathrm{~N}_{4} \mathrm{VO}_{3}$ & 541.143859 & 541.143902 & 78 & 0.12 \\
$\mathrm{C}_{31} \mathrm{H}_{28} \mathrm{~N}_{4} \mathrm{VO}_{3}$ & 555.159713 & 555.159552 & -29 & 0.25 \\
$\mathrm{C}_{32} \mathrm{H}_{30} \mathrm{~N}_{4} \mathrm{VO}_{3}$ & 569.175158 & 569.175202 & 78 & 0.58 \\
$\mathrm{C}_{33} \mathrm{H}_{32} \mathrm{~N}_{4} \mathrm{VO}_{3}$ & 583.190906 & 583.190852 & -93 & 0.22 \\
\hline
\end{tabular}

Table S4. Sulfur vanadyl porphyrin distribution in fraction $E_{6}$

\begin{tabular}{ccccc} 
KMD = 0.49512 & VOS Porphyrins “DBE = 22” & \multicolumn{2}{c}{ RMS = 106 ppb } & \\
\hline Molecular & $\begin{array}{c}\mathrm{m} / \mathbf{z} \\
\text { observed }\end{array}$ & $\begin{array}{c}\mathrm{m} / \mathbf{z} \\
\text { calculated }\end{array}$ & $\begin{array}{c}\text { Mass Error } \\
\text { ppb }\end{array}$ & $\begin{array}{c}\text { Relative } \\
\text { Abundance }\end{array}$ \\
\hline Formula & 565.126255 & 565.126143 & -197 & 0.16 \\
$\mathrm{C}_{32} \mathrm{H}_{26} \mathrm{~N}_{4}$ VOS & 579.141752 & 579.141793 & 72 & 0.20 \\
$\mathrm{C}_{33} \mathrm{H}_{28} \mathrm{~N}_{4}$ VOS & 593.15753 & 593.157444 & -146 & 0.33 \\
$\mathrm{C}_{34} \mathrm{H}_{30} \mathrm{~N}_{4}$ VOS & 607.173063 & 607.173094 & 50 & 0.21 \\
$\mathrm{C}_{35} \mathrm{H}_{32} \mathrm{~N}_{4}$ VOS & 621.188749 & 621.188744 & -08 & 0.19 \\
$\mathrm{C}_{36} \mathrm{H}_{34} \mathrm{~N}_{4}$ VOS & 635.204381 & 635.204394 & 21 & 0.13 \\
$\mathrm{C}_{37} \mathrm{H}_{36} \mathrm{~N}_{4}$ VOS & 649.220125 & 649.220044 & -125 & 0.19 \\
$\mathrm{C}_{38} \mathrm{H}_{38} \mathrm{~N}_{4}$ VOS & 663.235638 & 663.235694 & 84 & 0.12 \\
$\mathrm{C}_{39} \mathrm{H}_{40} \mathrm{~N}_{4}$ VOS & & & &
\end{tabular}




\begin{tabular}{ccccc} 
KMD = 0.48174 & VOS Porphyrins “DBE = 23” & RMS = 87 ppb & \\
\hline Molecular & $\begin{array}{c}\mathrm{m} / \mathbf{z} \\
\text { observed }\end{array}$ & $\begin{array}{c}\mathrm{m} / \mathbf{z} \\
\text { calculated }\end{array}$ & $\begin{array}{c}\text { Mass Error } \\
\text { ppb }\end{array}$ & $\begin{array}{c}\text { Relative } \\
\text { Abundance }\end{array}$ \\
\hline Formula & 577.126169 & 577.126143 & -45 & 0.11 \\
$\mathrm{C}_{33} \mathrm{H}_{24} \mathrm{~N}_{4}$ VOS & 591.141754 & 591.141793 & 67 & 0.13 \\
$\mathrm{C}_{34} \mathrm{H}_{26} \mathrm{~N}_{4}$ VOS & 605.15741 & 605.157444 & 56 & 0.14 \\
$\mathrm{C}_{35} \mathrm{H}_{28} \mathrm{~N}_{4}$ VOS & 619.173057 & 619.173094 & 59 & 0.22 \\
$\mathrm{C}_{36} \mathrm{H}_{30} \mathrm{~N}_{4}$ VOS & 633.18885 & 633.188744 & -168 & 0.31 \\
$\mathrm{C}_{37} \mathrm{H}_{32} \mathrm{~N}_{4}$ VOS & 647.204367 & 647.204394 & 42 & 0.42 \\
$\mathrm{C}_{38} \mathrm{H}_{34} \mathrm{~N}_{4}$ VOS & 661.220065 & 661.220044 & -32 & 0.27 \\
$\mathrm{C}_{39} \mathrm{H}_{36} \mathrm{~N}_{4}$ VOS & 675.235757 & 675.235694 & -93 & 0.19 \\
$\mathrm{C}_{40} \mathrm{H}_{38} \mathrm{~N}_{4}$ VOS & 689.251253 & 689.251344 & 133 & 0.15 \\
$\mathrm{C}_{41} \mathrm{H}_{40} \mathrm{~N}_{4}$ VOS & 703.267068 & 703.266994 & -105 & 0.22 \\
$\mathrm{C}_{42} \mathrm{H}_{42} \mathrm{~N}_{4}$ VOS & 717.282703 & 717.282644 & -82 & 0.18 \\
$\mathrm{C}_{43} \mathrm{H}_{44} \mathrm{~N}_{4}$ VOS & 731.29825 & 731.298294 & 60 & 0.11 \\
$\mathrm{C}_{44} \mathrm{H}_{46} \mathrm{~N}_{4}$ VOS & & &
\end{tabular}

\begin{tabular}{ccccc} 
KMD = 0.46833 & VOS Porphyrins “DBE = 24” & RMS = 158 ppb & \\
\hline Molecular & $\begin{array}{c}\mathrm{m} / \mathbf{z} \\
\text { observed }\end{array}$ & $\begin{array}{c}\mathrm{m} / \mathbf{z} \\
\text { calculated }\end{array}$ & $\begin{array}{c}\text { Mass Error } \\
\text { ppb }\end{array}$ & $\begin{array}{c}\text { Relative } \\
\text { Abundance }\end{array}$ \\
\hline Formula & 575.11056 & 575.110493 & -116 & 0.13 \\
$\mathrm{C}_{33} \mathrm{H}_{24} \mathrm{~N}_{4}$ VOS & 589.126247 & 589.126143 & -175 & 0.15 \\
$\mathrm{C}_{34} \mathrm{H}_{26} \mathrm{~N}_{4}$ VOS & 603.14186 & 603.141793 & -110 & 0.27 \\
$\mathrm{C}_{35} \mathrm{H}_{28} \mathrm{~N}_{4}$ VOS & 617.157484 & 617.157444 & -65 & 0.37 \\
$\mathrm{C}_{36} \mathrm{H}_{30} \mathrm{~N}_{4}$ VOS & 631.173115 & 631.173094 & -35 & 0.41 \\
$\mathrm{C}_{37} \mathrm{H}_{32} \mathrm{~N}_{4}$ VOS & 645.188749 & 645.188744 & -8 & 0.38 \\
$\mathrm{C}_{38} \mathrm{H}_{34} \mathrm{~N}_{4}$ VOS & 659.204547 & 659.204394 & -232 & 0.30 \\
$\mathrm{C}_{39} \mathrm{H}_{36} \mathrm{~N}_{4}$ VOS & 673.22014 & 673.220044 & -142 & 0.23 \\
$\mathrm{C}_{40} \mathrm{H}_{38} \mathrm{~N}_{4}$ VOS & 687.235723 & 687.235694 & -42 & 0.24 \\
$\mathrm{C}_{41} \mathrm{H}_{40} \mathrm{~N}_{4}$ VOS & 701.251281 & 701.251344 & 90 & 0.17 \\
$\mathrm{C}_{42} \mathrm{H}_{42} \mathrm{~N}_{4}$ VOS & 715.266881 & 715.266994 & 158 & 0.13 \\
$\mathrm{C}_{43} \mathrm{H}_{44} \mathrm{~N}_{4}$ VOS & 729.282647 & 729.282644 & -50 & 0.12 \\
$\mathrm{C}_{44} \mathrm{H}_{46} \mathrm{~N}_{4}$ VOS & 743.298001 & 743.298294 & 395 & 0.10 \\
$\mathrm{C}_{45} \mathrm{H}_{48} \mathrm{~N}_{4}$ VOS & & &
\end{tabular}

\begin{tabular}{ccccc} 
KMD = $\mathbf{0 . 4 5 4 9 4}$ & VOS Porphyrins “DBE = 25” & \multicolumn{2}{c}{ RMS = 180 ppb } & \\
\hline Molecular & $\begin{array}{c}\mathrm{m} / \mathbf{z} \\
\text { observed }\end{array}$ & $\begin{array}{c}\mathrm{m} / \mathbf{z} \\
\text { calculated }\end{array}$ & $\begin{array}{c}\text { Mass Error } \\
\text { ppb }\end{array}$ & $\begin{array}{c}\text { Relative } \\
\text { Abundance }\end{array}$ \\
\hline Formula & 587.110464 & 587.110493 & 51 & 0.10 \\
\hline $\mathrm{C}_{34} \mathrm{H}_{24} \mathrm{~N}_{4}$ VOS & 601.126151 & 601.126143 & -13 & 0.14 \\
$\mathrm{C}_{35} \mathrm{H}_{26} \mathrm{~N}_{4}$ VOS & 615.141845 & 615.141793 & -84 & 0.19 \\
$\mathrm{C}_{36} \mathrm{H}_{28} \mathrm{~N}_{4}$ VOS & 629.157497 & 629.157444 & -84 & 0.21 \\
$\mathrm{C}_{37} \mathrm{H}_{30} \mathrm{~N}_{4}$ VOS & & &
\end{tabular}




\begin{tabular}{|c|c|c|c|c|}
\hline $\mathrm{C}_{38} \mathrm{H}_{32} \mathrm{~N}_{4}$ VOS & 643.173176 & 643.173094 & -128 & 0.28 \\
\hline $\mathrm{C}_{39} \mathrm{H}_{34} \mathrm{~N}_{4}$ VOS & 657.188688 & 657.188744 & 85 & 0.29 \\
\hline $\mathrm{C}_{40} \mathrm{H}_{36} \mathrm{~N}_{4}$ VOS & 671.204393 & 671.204394 & 1 & 0.21 \\
\hline $\mathrm{C}_{41} \mathrm{H}_{38} \mathrm{~N}_{4}$ VOS & 685.220242 & 685.220044 & -29 & 0.19 \\
\hline $\mathrm{C}_{42} \mathrm{H}_{40} \mathrm{~N}_{4}$ VOS & 699.235672 & 699.235694 & 31 & 0.17 \\
\hline $\mathrm{C}_{43} \mathrm{H}_{42} \mathrm{~N}_{4}$ VOS & 713.251279 & 713.251344 & 91 & 0.16 \\
\hline $\mathrm{C}_{44} \mathrm{H}_{44} \mathrm{~N}_{4}$ VOS & 727.267174 & 727.266994 & -248 & 0.14 \\
\hline $\mathrm{C}_{45} \mathrm{H}_{46} \mathrm{~N}_{4}$ VOS & 741.282844 & 741.282644 & -27 & 0.09 \\
\hline KMD = 0.44174 & VOS Porphyrins & DBE = 26” & RMS = 118 ppb & \\
\hline $\begin{array}{c}\text { Molecular } \\
\text { Formula } \\
\end{array}$ & $\begin{array}{c}\mathrm{m} / \mathrm{z} \\
\text { observed }\end{array}$ & $\begin{array}{c}\mathrm{m} / \mathrm{z} \\
\text { calculated }\end{array}$ & $\begin{array}{l}\text { Mass Error } \\
\text { ppb }\end{array}$ & $\begin{array}{c}\text { Relative } \\
\text { Abundance }\end{array}$ \\
\hline $\mathrm{C}_{37} \mathrm{H}_{28} \mathrm{~N}_{4}$ VOS & 627.141829 & 627.141793 & -0.035 & 0.14 \\
\hline $\mathrm{C}_{38} \mathrm{H}_{30} \mathrm{~N}_{4}$ VOS & 641.157442 & 641.157444 & 0.001 & 0.16 \\
\hline $\mathrm{C}_{39} \mathrm{H}_{32} \mathrm{~N}_{4}$ VOS & 655.173132 & 655.173094 & -0.038 & 0.21 \\
\hline $\mathrm{C}_{40} \mathrm{H}_{34} \mathrm{~N}_{4}$ VOS & 669.188707 & 669.188744 & 0.037 & 0.34 \\
\hline $\mathrm{C}_{41} \mathrm{H}_{36} \mathrm{~N}_{4}$ VOS & 683.204388 & 683.204394 & 0.006 & 0.22 \\
\hline $\mathrm{C}_{42} \mathrm{H}_{38} \mathrm{~N}_{4}$ VOS & 697.220079 & 697.220044 & -0.036 & 0.13 \\
\hline $\mathrm{C}_{43} \mathrm{H}_{40} \mathrm{~N}_{4}$ VOS & 711.235543 & 711.235694 & 0.151 & 0.11 \\
\hline $\mathrm{C}_{44} \mathrm{H}_{42} \mathrm{~N}_{4}$ VOS & 725.251512 & 725.251344 & -0.168 & 0.10 \\
\hline
\end{tabular}

